\title{
Thermophoretic Manipulation of Micro- and Nanoparticle Flow through a Sudden Contraction in a Microchannel with Near-Infrared Laser Irradiation
}

\author{
Tetsuro Tsuji, ${ }^{*}$ Yuta Sasai, and Satoyuki Kawano ${ }^{\dagger}$ \\ Graduate School of Engineering Science, Osaka University, 1-3 Machikaneyama, Toyonaka, Osaka 560-8531, \\ Japan
}

(Received 11 May 2018; revised manuscript received 7 August 2018; published 2 October 2018)

\begin{abstract}
A temperature gradient in a continuous fluid induces the motion of dispersed micro- and nanoparticles even when the fluid is motionless. This phenomenon is known as thermophoresis, and it is expected to be the basis for techniques to control particle motion. In this study, we use the thermophoresis of microand nanoparticles in a microchannel filled with an aqueous solution to control the particle motion near the inlet of a sudden contraction, which is a narrower channel connecting two wider channels. Microfluidic devices with sudden contractions are widely used to develop various devices with micro- and nanometer dimensions, such as nanopore sensors. A near-infrared laser is used to create a strong temperature gradient of $O\left(10^{6}\right) \mathrm{K} \mathrm{m}^{-1}$ and induce thermophoresis of micro- and nanoparticles. Because the heating by the laser irradiation is localized near the inlet of the contraction, this configuration is useful for controlling particle translocation into and through the contraction. We characterize our experimental setup by quantifying flow and temperature fields near the contraction channel using particle image velocimetry and laser-induced fluorescence, respectively. Then, we observe the obstruction of the particle translocation into the contraction channel induced by the laser-induced thermophoresis for various parameters such as channel dimensions, flow speeds, particle sizes, and laser powers. Near the inlet of the contraction channel, the counterbalance of thermophoretic force and flow drag leads to the ringlike pattern formation of the particle distribution. Moreover, we carry out some demonstrations using the proposed system to selectively translocate particles and enhance the sensing performance due to increased particle density. Thanks to the noncontact nature of laser-induced thermophoresis, the integration of our method into existing microfluidic devices is feasible and expected to improve technologies for manipulating particles in fluids.
\end{abstract}

DOI: 10.1103/PhysRevApplied.10.044005

\section{INTRODUCTION}

Transport of molecules and micro- and nanoparticles in confined spaces such as micro- and nanofluidic channels is widely exploited in the development of lab-on-achip devices for biomedical applications [1]. In particular, sudden contractions (i.e., abrupt constrictions) are often fabricated in these devices [2-11] to equip them with narrower channels, which are connected to wider channels, with specific functions such as sensing or filtering. Because the target objects usually have excess electric charge in a solution, most devices use electrokinetic approaches $[2,4-7,12]$; that is, a voltage drop is applied through the fluid channels to control the motion of small objects in the

\footnotetext{
*tsuji@me.es.osaka-u.ac.jp

†kawano@me.es.osaka-u.ac.jp

Published by the American Physical Society under the terms of the Creative Commons Attribution 4.0 International license. Further distribution of this work must maintain attribution to the author(s) and the published article's title, journal citation, and DOI.
}

narrower channels. Such an approach works well because the contraction geometrically enhances the nonuniformity of the electric potential by concentrating the lines of electric force, which is necessary for electrophoretic or dielectrophoretic manipulation of the targets. However, if a mixture of particles with similar electric properties is considered (e.g., similar sizes and surface charge densities), the electrokinetic approach is insufficient for achieving selective particle manipulation. In the present paper, we propose an alternative manipulation technique based on the nonuniformity of the temperature field of a solution and give additional parameters toward the selective manipulation of micro- and nanoparticles.

A temperature gradient in a continuous solution induces motion in dispersed target objects even when the solution is motionless. Such a transport phenomenon is called thermophoresis as reviewed in Refs. [13-15]. Thermophoresis has been investigated to manipulate biomolecules [16-22] and cells [23], as well as colloids for more fundamental investigations [24-40]. It can also be applied to the concentration of DNA [41] or the measurement of protein binding $[42,43]$. An interesting point of thermophoretic 
(a)

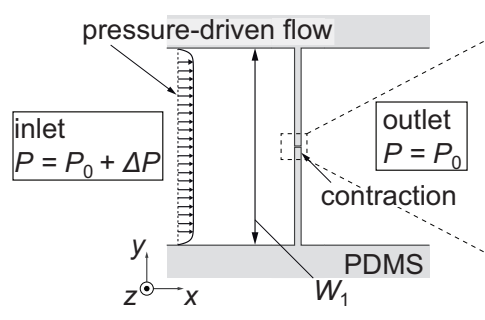

magnified view



(b)

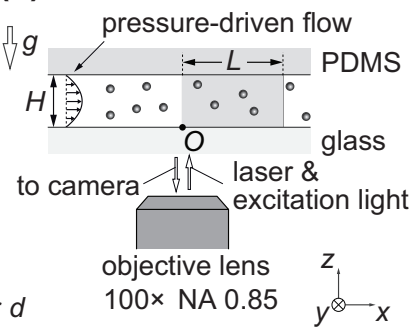

FIG. 1. Overview of experiments. (a) Top view of a microchannel with width $W_{1}=1.5 \mathrm{~mm}$ and height $H$ that has a sudden narrowing to width $W=10 \mu \mathrm{m}$ and length $L$. Height $H$ and length $L$ are summarized in Table I. A pressure difference between the inlet and outlet is maintained at $\Delta P$, resulting in a steady flow of a sample solution containing particles through the microchannel. (b) Side view (section $\mathrm{A}-\mathrm{A}^{\prime}$ ) of the experimental setup. The microchannel is observed from underneath with a microscope objective lens. The sample solution is irradiated by laser and fluorescence excitation light through the same lens.

manipulation, although its mechanism has not been fully understood yet, arises from the fact that the thermophoretic characteristics are sensitive to the physical properties of target objects and the solvent in which they are immersed. In other words, the direction of thermophoretic motion can be tuned independently of electrophoretic or dielectrophoretic characteristics, such as surface charge density or electric permittivity. Theoretical studies to elucidate thermophoretic characteristics have been conducted using molecular dynamics simulation [44-46], mesoscale simulation [47], and hydrodynamic or kinetic approaches [15,48-52].

Given such a growing interest in thermophoretic manipulation, some fundamental characterizations of thermophoresis near the contraction channels are necessary for further improvement of the manipulation technique. This study aims to carry out a systematic characterization of thermophoresis for translocating micro- and nanoparticles through contractions based on our previous study of thermophoresis in a microchannel [39]. We use a nearinfrared laser focused on the entrance of the contraction channel to heat the solution locally. The laser heating cre-

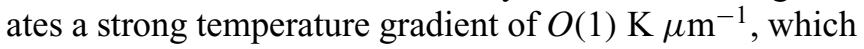
induces the thermophoresis of micro- and nanoparticles near the contraction and thus controls the particle motion there. We further carry out some demonstrations using the proposed method to show selective particle translocation and enhancement of sensing performance due to increased particle density. Such demonstrations reveal a noncontact method with simple fabrication that can be integrated into existing microfluidic devices easily and can improve particle-velocity control in micro- and nanoscale channels.

Finally, we close this section by introducing to readers one successful application of a microfluidic channel with a sudden contraction, a nanopore sensor. The device uses electrical sensing to detect and/or identify polymers or larger biomolecules, such as DNA bases [53-59], by translocating them into the nanopore part, which can be considered as the sudden contraction. In the detection process, the velocities of targets through the nanopore are important because the velocity-control performance is directly related to the sensor accuracy. Various velocity-control techniques have been applied inside the nanopore [60-62]: tethering a protein larger than the nanopore diameter to prevent fast translocation $[63,64]$, coating nanopore walls with counteractive charges $[65,66]$ or polymers [67], using active feedback control with an applied voltage [68], decreasing the temperature to increase the viscosity of the solvent [69], and inplane guiding of targets via dielectrophoresis by AC electric fields at the nanopore entrance [70]. However, most of these techniques need highly sophisticated fabrication apparatuses and experimental skills. These difficulties usually result in a challenging trial-and-error process, a low yield rate, and widely varying device performance. Therefore, alternative control methods are needed to achieve more feasible engineering applications. The use of thermophoresis to control the motion of a DNA base near nanopores has been proposed using numerical simulation [71-73] and a recent nanopore experiment [74,75]. We expect that the quantitative evaluation of thermophoreticmanipulation characteristics near a sudden-contraction microchannel, as performed in this study, will be a reference study toward the design of nanopore sensors.

\section{EXPERIMENTAL SETUP}

\section{A. Overview}

First, we provide an overview of the experiments, all of which are conducted at room temperature, specifically, $298.5 \pm 1.3 \mathrm{~K}$. We use a microchannel of width $W_{1}=1.5 \mathrm{~mm}$ and height $H$, which has a sudden contraction to a width $W=10 \mu \mathrm{m}$ and length $L$, as shown in Fig. 1. We investigate three microchannel configurations by changing the values of $H$ and $L$, as summarized in Table I in Sec. II B. The Cartesian coordinate system is defined with the origin placed at the entrance of the contraction. The microchannel is filled with an 
TABLE I. Dimensions of the microchannels and resolution of the flow control $\bar{u}_{x, \min }=\alpha \Delta P_{\text {min }}$.

\begin{tabular}{|c|c|c|c|c|}
\hline Device & & $L(\mu \mathrm{m})$ & $H(\mu \mathrm{m})$ & $\bar{u}_{x, \min }\left(\mu \mathrm{m} \mathrm{s}^{-1}\right)$ \\
\hline A & 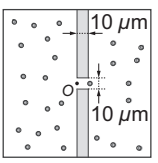 & 10 & $8.4 \pm 0.2$ & 1.1 \\
\hline B &  & 50 & $8.1 \pm 0.1$ & $4.2 \times 10^{-1}$ \\
\hline $\mathrm{C}$ & 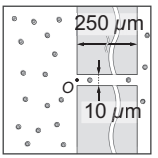 & 250 & $7.0 \pm 0.1$ & $9.4 \times 10^{-2}$ \\
\hline
\end{tabular}

aqueous solution containing particles with diameter $d$. The inlet and outlet are connected to reservoirs that are open and exposed to atmosphere. Thus, the pressure $P$ at the inlet and outlet is maintained at the atmospheric pressure $P_{0}$. We induce a pressure-driven flow in the microchannel by lifting the inlet chamber by $\Delta h$ using a microstage (HPS60-20X, Sigma Koki, Tokyo, Japan) with a resolution of $\Delta h_{\min }=1 \mu \mathrm{m}$. In this way, a water-level difference $\Delta h$ is generated between the inlet and outlet chambers. In other words, the total pressure $P$ at the inlet is given by $P=P_{0}+\Delta P$, where $\Delta P=\rho g \Delta h$, in which $\rho=10^{3} \mathrm{~kg} \mathrm{~m}^{-3}$ is the density of water and $g=9.8 \mathrm{~m} \mathrm{~s}^{-2}$ is the acceleration of gravity. Note that the above setup can control the pressure difference with an accuracy of $\Delta P=\Delta P_{\min } \approx 10^{-2} \mathrm{~Pa}$.

As shown in Fig. 1(b), the microchannel is observed from underneath through an objective lens $(\times 100$ magnification, NA $=0.85$, LCPLN100XIR, Olympus, Tokyo, Japan) and images are recorded with a CMOS camera (Zyla 5.5, Andor Technology, Belfast, Northern Ireland). As described in Sec. II C, the laser and excitation light are transmitted through the same objective lens. The laser's purpose is to induce a temperature increase and the excitation light enables fluorescence observation of particle movement and position. The optical axis of the laser is along the $z$ direction and is set at the origin as shown in Fig. 1(b).

\section{B. Microfluidic device}

The microfluidic device used here is fabricated by bonding a polydimethylsiloxane (PDMS) block (SYLGARD ${ }^{\circledR}$ 184, Dow Corning Toray Co., Ltd, Tokyo, Japan) and a glass substrate $\left(24 \times 36 \mathrm{~mm}^{2}\right.$ No. 1 , Matsunami Glass Co. Ltd., Osaka, Japan). Before bonding, the surfaces of the PDMS block and the glass substrate are cleaned with a corona treatment (BD-20AC, Electro-Technic Products, Chicago, USA) to enhance the adhesion. The wider parts of the microchannel with width $W_{1}=1.5 \mathrm{~mm}$ are prone to collapse because of their low aspect ratio, that is, $H / W_{1}<$ $6 \times 10^{-3}$. To avoid this collapse, cylindrical arrays made of PDMS, which are not shown in Fig. 1(a), are placed as columns in the wider parts of the microchannel. The diameter of each column is $100 \mu \mathrm{m}$; the distance between the column centers is $400 \mu \mathrm{m}$; and the distances of the columns from the origin, which is placed at the inlet of the sudden contraction as shown in Fig. 1(b), are more than $300 \mu \mathrm{m}$. Therefore, all the columns are sufficiently distant from the test section, which is centered at the origin and has dimensions of $131 \times 131 \mu \mathrm{m}^{2}$. Their presence does not affect the flow behavior discussed in this paper.

The PDMS block is cast from a mold made from SU8 photoresist with a fluid channel pattern. Details of the fabrication of the SU-8 mold are described in our previous study [39]. The PDMS block has two holes for the inlet and outlet, which are made with a biopsy punch $(2.5 \mathrm{~mm}$, BP25F, Kai Industries Co., Ltd., Seki, Japan). Silicone tubes connect each of the holes to the inlet and outlet reservoirs, respectively. As described in Sec. II A, the water-level difference $\Delta h$ between solutions in these two reservoirs is regulated to control the flows in the microchannel. The pressure-driven flow is assumed to have a steady state because the reservoirs have cross-sectional areas $A_{\text {res }}$ much larger than that of the contraction $A=W H$ (see Table I), that is, $A / A_{\text {res }}<10^{-6}$. For example, to produce a decrease of $\Delta h=\Delta h_{\min }=1 \mu \mathrm{m}$ with a typical flow speed of $10 \mu \mathrm{m} \mathrm{s}^{-1}$ through the contraction, it takes more than $50 \mathrm{~h}$, which is much more than our typical experimental duration of $5 \mathrm{~min}$.

We fabricate three configurations of microchannels and their dimensions are summarized in Table I. Device A has the smallest orifice structure, with $L=10 \mu \mathrm{m}$, while devices B and C are longer at $L=50 \mu \mathrm{m}$ and $L=250 \mu \mathrm{m}$, respectively. The height $H$ is measured by scanning the PDMS block using a laser displacement sensor (LKH008W, Keyence, Osaka, Japan). In the present experiments, the estimation and control of flow fields near the contraction channel is important because the flow velocity of the aqueous solution determines the device performance. We theoretically estimate the magnitude of the flow based on the methods in [76,77], which are useful for evaluating steady-state pressure drops over microchannels. The details of the analysis are given in Appendix A, but we can summarize the obtained results as follows. The mean flow velocity $\bar{u}_{x}$ in the $x$ direction is calculated as $\bar{u}_{x}=\alpha \Delta P$ in the contraction, where $\alpha\left(\mathrm{m} \mathrm{s}^{-1} \mathrm{~Pa}^{-1}\right)$ is the sensitivity of $\bar{u}_{x}$ to $\Delta P$. Therefore, $\bar{u}_{x, \min }=\alpha \Delta P_{\min }$ is the resolution of the control of the flow speed in the contraction channel. The values of $\bar{u}_{x, \min }$ are also presented in Table I. We can see that device A with smaller $L$ has worse resolution $\bar{u}_{x, \text { min }}$ for flow control. That is, it is easier to precisely control the flow in device $\mathrm{C}$ and this design is used most often in our study. 


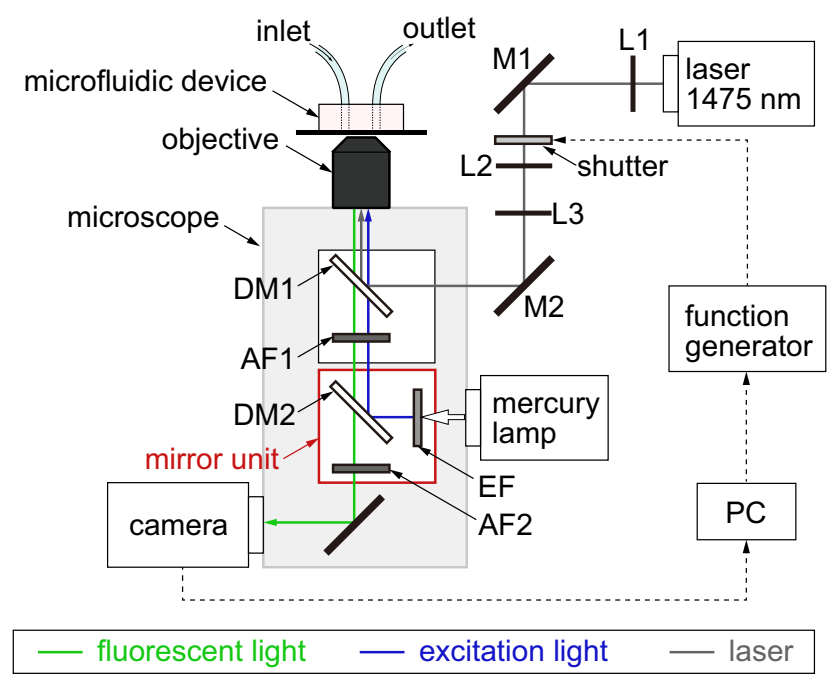

FIG. 2. Optical setup for laser irradiation and fluorescence observation. Sample solutions in microfluidic devices are exposed to a laser with a wavelength of $1475 \mathrm{~nm}$ and a fluorescence excitation light through the objective lens. The camera detects only the fluorescent light from sample solutions. DM1 denotes the dichroic mirror to combine the laser with the excitation light; DM2, the dichroic mirror to separate the excitation light from the emitted fluorescent light; AF1, the absorption filter for the laser; AF2, the absorption filter for the excitation light; EF, the excitation filter; L1, L2, and L3, the planoconvex lenses for laser shaping; M1 and M2, the mirrors for laser alignment. PC, the personal computer to analyze the images obtained by the camera and synchronize the image acquisition and laser irradiation. Appropriate mirror units are used according to the excitation/fluorescence wavelength of the sample.

\section{Optical setup}

The optical setup for laser irradiation and fluorescence observation is illustrated in Fig. 2. A laser with a wavelength of $\lambda=1475 \mathrm{~nm}$ is used. The absorption coefficient $\beta_{\mathrm{abs}}$ of $\lambda=1475 \mathrm{~nm}$ for an aqueous solution can be estimated as $\beta_{\mathrm{abs}}=2.5 \times 10^{3} \mathrm{~m}^{-1}$ [78-80]. This value of $\beta_{\mathrm{abs}}$ is significantly greater than that commonly used for visible or infrared lasers; for example, $\beta_{\mathrm{abs}}=4 \times 10^{-2} \mathrm{~m}^{-1}$ and $\beta_{\text {abs }}=11 \mathrm{~m}^{-1}$ are obtained for $\lambda=532 \mathrm{~nm}$ and $\lambda=$ $1064 \mathrm{~nm}$, respectively. The energy $\dot{q}$ per unit time and per unit volume absorbed by the aqueous solution is described as $\dot{q}=\beta_{\text {abs }} I$, where $I\left(\mathrm{~W} \mathrm{~m}^{-2}\right)$ is the irradiated laser intensity and, thus, a laser with $\lambda=1475 \mathrm{~nm}$ is strongly absorbed by the water compared with lasers with shorter wavelengths.

A continuous infrared laser with $\lambda=1475 \mathrm{~nm}$ (FPLB1475, FiberLabs Inc., Fujimino, Japan) is collimated and shaped through planoconvex lenses, as shown in Fig. 2. The laser power after the objective lens is measured by a power meter (3A-QUAD, Ophir Optronics, Jerusalem, Israel). The positions of the planoconvex lenses are adjusted to maximize the laser power after the objective lens. In our optical setup, the laser power after the objective lens is $47.2 \pm 0.3 \%$ of that at the laser driver. The laser is combined with excitation light from a mercury lamp (U-HGLGPS, Olympus, Tokyo, Japan) using a dichroic mirror (DM1) in the microscope and it irradiates the sample solution in the microchannel through the objective lens. Fluorescent light emitted by the sample solution is separated from other light by a dichroic mirror (DM2) and recorded by the camera. The obtained images are analyzed by a personal computer. A trigger signal from the camera is used to control the shutter between the laser and sample. Thus, the observation and the laser irradiation are synchronized.

\section{RESULTS AND DISCUSSION}

\section{A. Evaluation of flow and temperature fields \\ 1. Flow fields}

We use fluorescent polystyrene (PS) particles $(d=$ $0.99 \pm 0.022 \mu \mathrm{m}, \mathrm{F} 8823$, Molecular Probes, Eugene, USA) as tracers and carry out particle-image velocimetry (PIV) (Flownizer 2D, Detect Co. Ltd., Tokyo, Japan) of video images obtained at 20 frames per second. Note that the motion of particles represents the flow field because no laser irradiation is used in the experiment described in this section. Figure 3(a) shows a typical result for the flow velocity field $\mathbf{u}$ and its magnitude $|\mathbf{u}|$, where device $\mathrm{C}$ is used and $\Delta P$ is $1.0 \pm 0.01 \mathrm{~Pa}$. Various reference vectors are used for $x<0 \mu \mathrm{m}$ and $x>0 \mu \mathrm{m}$. For $x<0 \mu \mathrm{m}$, flow toward the contraction is observed and the flow speed increases as $r=\sqrt{x^{2}+y^{2}}$ decreases. At $x>0 \mu \mathrm{m}$, the flow speed reaches $10 \mu \mathrm{m} \mathrm{s}^{-1}$. Similar velocity fields are obtained for devices $\mathrm{A}$ and $\mathrm{B}$ with different values for $\Delta P$. In Table II, we summarize typical values obtained using PIV analysis. The mean flows through the contraction $\bar{u}_{x}$ are compared between theory and experiment for various values of $\Delta P$. It is seen that devices $\mathrm{A}$ and $\mathrm{B}$ produce poor consistencies between the theory and experiment; namely, theoretical values overestimate the mean flow and relative errors are $16 \%-63 \%$. This overestimation by the theory may be caused by the assumption, described in Appendix $\mathrm{A}$, that minor losses can be ignored. This assumption may not be valid for devices A and B with short channel length $L$. In contrast, device $\mathrm{C}$ yields better results with relative errors less than $6 \%$. This is due to the fact that device $\mathrm{C}$ has better flow-control ability, as discussed in Sec. II B. In Fig. 3(b), the flow velocity profile in the $x$ direction, $u_{x}=$ $u_{x}(y)$, averaged over the rectangular region in Fig. 3(c) in the contraction, is presented for $\Delta P=10 \mathrm{~Pa}$. The dashed curve in Fig. 3(b) is the fitting by the Poiseuille flow in a rectangular channel [81] averaged over the $z$ direction. We conclude that the flow velocity measurement is well predicted by the Poiseuille-type flow for device C. It should be noted that, near the wall at $y= \pm 5 \mu \mathrm{m}$, the experimental 

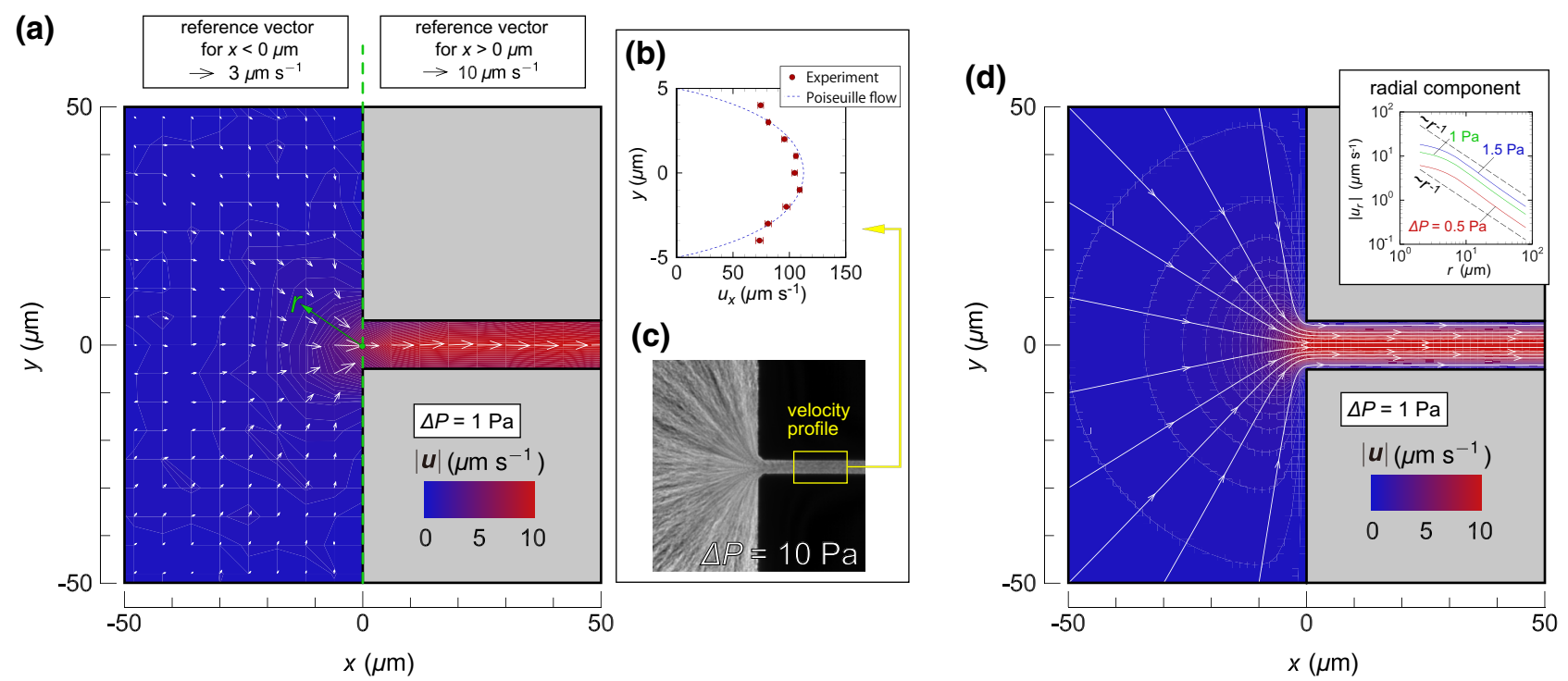

FIG. 3. (a) Experimental results of flow velocity field $\mathbf{u}$ in device $\mathrm{C}$ with $\Delta P=1 \mathrm{~Pa}$. Note that different reference vectors are used for $x<0 \mu \mathrm{m}$ and $x>0 \mu \mathrm{m}$. For $x<0 \mu \mathrm{m}$, flow toward the contraction is observed and the flow speed increases as $r=\sqrt{x^{2}+y^{2}}$ decreases. For $x>0 \mu \mathrm{m}$, the flow speed reaches $10 \mu \mathrm{m} \mathrm{s}^{-1}$ in the contraction. (b) Flow velocity profile $u_{x}=u_{x}(y)$ in the contraction for $\Delta P=10 \mathrm{~Pa}$. (c) The superposition of the snapshots for $\Delta P=10 \mathrm{~Pa}$. Stream lines are visualized by the trajectories of the tracer particles. (d) Simulation results of stream lines and flow speeds for the same conditions as in panel (a). The inset shows the radial component of the flow velocity $\left|u_{r}\right|$ as a function of $r . u_{r}$ is well approximated by the function $r^{-1}$ for $r>10 \mu \mathrm{m}$.

results are slightly larger than those of the Poiseuille-type flow profile. In micro-PIV measurement, such a deviation was reported previously (e.g., Ref. [82]), where the error arises due to the low numerical aperture (NA) of our imaging system, Brownian motion, or nonuniform particle distribution in the cross section ( $y-z$ plane).

We also present the flow speed for the wider channel, that is, $x<0$. Figure 3(c) shows the superposition of the snapshots for $\Delta P=10 \mathrm{~Pa}$. From this figure, it is seen that the velocity of particles, that is, the flow vector, has only the $r$ component except near the origin. This flow field can be explained as follows. We choose a length scale larger than the size of the contraction, $W=10 \mu \mathrm{m}$, and

TABLE II. Relation between the pressure difference $\Delta P$ and the flow speed $\bar{u}_{x}$ in the contraction and the radial flow velocity component $u_{r}$ for devices A, B, and C.

\begin{tabular}{lccccc}
\hline \hline & $\begin{array}{c}\Delta P \\
\text { Device }\end{array}$ & $\begin{array}{c}\bar{u}_{x}\left(\mu \mathrm{m} \mathrm{s}^{-1}\right) \\
(\text { theory })\end{array}$ & $\begin{array}{c}\bar{u}_{x}\left(\mu \mathrm{m} \mathrm{s}^{-1}\right) \\
(\text { experiment })\end{array}$ & $\begin{array}{c}\text { Relative } \\
\text { error }(\%)\end{array}$ & $\begin{array}{c}\left.u_{r}\right|_{r=20 \mu \mathrm{m}} \\
\left(\mu \mathrm{m} \mathrm{s}^{-1}\right)\end{array}$ \\
\hline A & 0.1 & 8.66 & $6.09 \pm 1.0$ & 30 & -1.7 \\
& 0.2 & 17.3 & $7.75 \pm 0.98$ & 55 & -3.1 \\
& 0.5 & 43.3 & $15.6 \pm 1.60$ & 63 & -6.8 \\
$\mathrm{~B}$ & 0.1 & 3.79 & $1.68 \pm 0.49$ & 56 & -0.25 \\
& 0.2 & 7.57 & $5.63 \pm 0.87$ & 26 & -1.1 \\
& 0.5 & 18.9 & $15.9 \pm 0.83$ & 16 & -2.1 \\
$\mathrm{C}$ & 0.5 & 4.66 & $4.72 \pm 0.87$ & 1 & -0.82 \\
& 1.0 & 9.33 & $9.48 \pm 0.89$ & 2 & -1.27 \\
& 1.5 & 14.0 & $14.8 \pm 1.13$ & 6 & -1.92 \\
\hline \hline
\end{tabular}

treat the contraction as a point sink source. As described in Appendix A, the flow is incompressible, Newtonian, and steady with a low Reynolds number (Re). In the wider channel, except at the entrance of the contraction, the spatial length scale in the $z$ direction $(H<10 \mu \mathrm{m})$ is significantly smaller than that in the $x$ or $y$ direction (approximately $W_{1}=1.5 \mathrm{~mm}$ ). Therefore, the flow field varies slowly in the $x$ and $y$ directions. Then, we are allowed to assume that $\partial^{2} \mathbf{u} / \partial x^{2} \approx \partial^{2} \mathbf{u} / \partial y^{2} \ll \partial^{2} \mathbf{u} / \partial z^{2}$ and the Navier-Stokes equation without inertia terms can be written as

$$
\begin{gathered}
0=-\frac{\partial p}{\partial x}+v \frac{\partial^{2} u_{x}}{\partial z^{2}}, \\
0=-\frac{\partial p}{\partial y}+v \frac{\partial^{2} u_{y}}{\partial z^{2}}, \\
0=-\frac{\partial p}{\partial z} \\
\frac{\partial u_{x}}{\partial x}+\frac{\partial u_{y}}{\partial y}=0,
\end{gathered}
$$

where $v(=\eta / \rho)$ is kinematic viscosity and $p$ is the pressure field. Equation (1c) leads to $p=p(x, y)$. Then, Eqs. (1a) and (1b) can be integrated with respect to $z$, yielding the flow fields as $u_{x}=(1 / 2 v)(\partial p / \partial x) z(z-H)$ and $u_{y}=(1 / 2 v)(\partial p / \partial y) z(z-H)$. With the aid of the equation of continuity (1d), the summation of Eqs. (1a) and $(1 \mathrm{~b})$ results in the Poisson equation of the pressure field: $\partial^{2} p / \partial x^{2}+\partial^{2} p / \partial y^{2}=0$. The Poisson equation has 


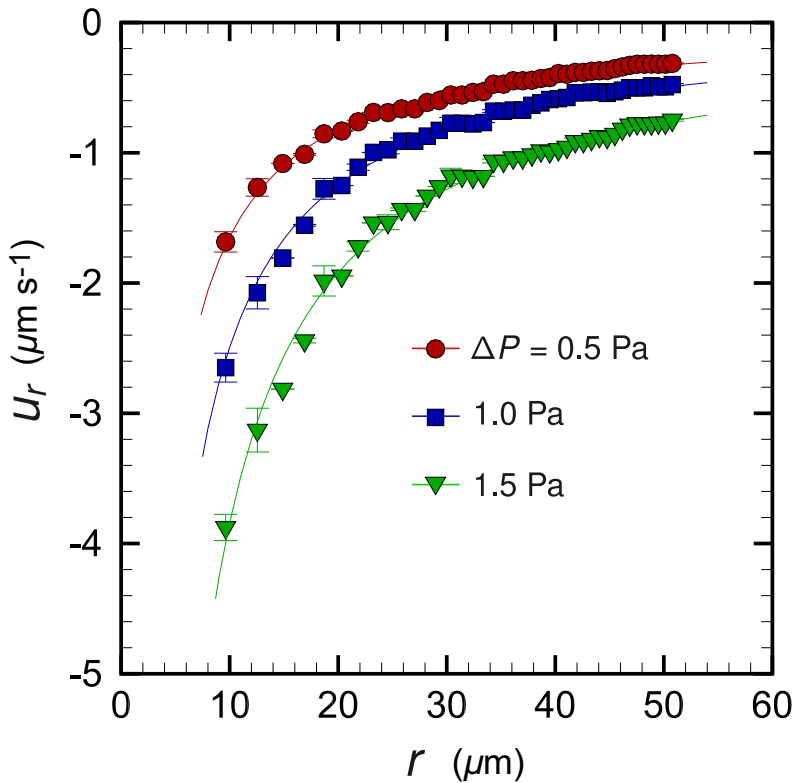

FIG. 4. Flow velocity component in the radial direction $u_{r}(r)$, where $r=\sqrt{x^{2}+y^{2}}$ for $\Delta P=0.5,1.0$, and $1.5 \mathrm{~Pa}$ in device $\mathrm{C}$. Fitting curves are $-C_{r} r^{-1}$, where $C_{r}$ is a fitting parameter.

a solution dependent only on the $r$ direction, $p=-\log r$. Substitution of this pressure field into the expressions of $u_{x}$ and $u_{y}$ leads to a flow field that is proportional to $-f(z) / r$, where $f(z)=z(z-H) / 2 v$. The radial flow velocity component is defined as $u_{r}$ and $u_{r}$ at $r=\sqrt{x^{2}+y^{2}}=20 \mu \mathrm{m}$ is shown in Table II. It is seen that $\left|u_{r}\right|$ increases in step with $\Delta P$. The flow fields obtained by numerical analysis using the commercial code COMSOL MULTIPHYSICS 5.2 (COMSOL, Inc., Stockholm, Sweden) are described in Fig. 3(d). For device C, the overall flow field and the dependence of $r^{-1}$ on $\left|u_{r}\right|$ is well reproduced. In our experiments, $\left|u_{r}\right|=O(1) \mu \mathrm{m} \mathrm{s}^{-1}$ for all cases and $\left|u_{r}\right|$ has the same order of magnitude as the thermophoretic drift speed $D_{T}|\nabla T|$ obtained by the appropriate order of $D_{T}$ for microand nanoparticles in liquid, $D_{T}=1 \sim 10 \mu \mathrm{m}^{2} \mathrm{~s}^{-1} \mathrm{~K}^{-1}$ $[13,14]$, and the temperature gradient $|\nabla T|=0.5{\mathrm{~K} \mu \mathrm{m}^{-1}}^{-1}$ [39]. Note that the actual value of $D_{T}$ is system specific but the order of magnitude is rather universal and within the above range [13]. The radial flow velocity component $u_{r}$ in device $\mathrm{C}$ is presented for $\Delta P=0.5,1.0$, and $1.5 \mathrm{~Pa}$ in Fig. 4. Fitting curves are obtained as $-C_{r} / r$, where $C_{r}$ is a fitting parameter. This functional form is derived from the above discussion using the Navier-Stokes equation. The fitting curves are used in Sec. III B to estimate thermophoretic mobility $D_{T}$.

\section{Temperature fields}

Nonuniform temperature fields of aqueous solutions are produced by focusing the laser on the microchannel by the method described in Sec. IIC. The temperature fields are measured using the laser-induced fluorescence (LIF) method $[30,39,83]$. A mixed solution of the fluorescent molecule 2',7'-bis-(2-carboxyethyl)-5-(6)carboxyfluorescein (BCECF) (Molecular Probes, Eugene, USA) and tris(hydroxymethyl)amino methane hydrochloride (tris- $\mathrm{HCl})(\mathrm{pH}=8.0$, Nippon Gene, Tokyo, Japan) is used as the temperature-sensitive sample. The concentrations of BCECF and tris- $\mathrm{HCl}$ are $20 \mu \mathrm{mol} \mathrm{l}^{-1}$ and $10 \mathrm{mmol} \mathrm{1}^{-1}$, respectively. It should be noted that the present microfluidic device is thin, with a channel height less than $10 \mu \mathrm{m}$, so the obtained temperature fields are the average temperature in the $z$ direction. It is qualitatively confirmed by numerical analysis using comsol multiphysics 5.2 that the effect of natural convection on the particle motion is negligible and omitted from the results presented here. The average temperature field is denoted by $T$ in the following. We add a small amount of tracer particles $\left(10^{-2} \mathrm{wt} \%, d=1.0 \pm 0.028 \mu \mathrm{m} ; \mathrm{F} 8821\right.$, Molecular Probes, Eugene, USA), which have fluorescence characteristics different from BCECF, so only BCECF can be observed by choosing an appropriate mirror unit (UFBW, Olympus, Tokyo, Japan) and only the tracer particles can be observed with a different mirror unit (U-FGW, Olympus, Tokyo, Japan).

First, we observe the tracer particles to ensure that flow in the microchannel is not induced; that is, we confirm that $\Delta P=0 \mathrm{~Pa}$. Then, we apply the pressure difference $\Delta P$ and induce flow in the microchannel. Subsequently, we change the mirror unit to observe only the fluorescence of BCECF. As discussed in Ref. [39], the temperature field rapidly approaches the steady state in a couple of seconds. We confirm that the steady state in the present study is established $1 \mathrm{~s}$ after the start of laser irradiation, as shown later in Fig. 5(c). The subsequent images for $3 \mathrm{~s}$ are analyzed and averaged to obtain the temperature field presented subsequently. We use device $\mathrm{C}$ for the results presented in this section.

Figure 5(a) shows the maximum temperature $T_{\max }=$ $\max _{0 \leq r \leq 60 \mu \mathrm{m}} T$ and radial temperature gradient $\partial T / \partial r$ induced by laser irradiation for $\Delta P=0 \mathrm{~Pa}$. Note that the temperature gradient is evaluated at $r=r_{\max }=7.7 \pm$ $1.4 \mu \mathrm{m}$, at which the magnitude of temperature gradient $|\partial T / \partial r|$ is maximum. The experiments in this study are conducted by irradiating the entrance of the contraction with the laser when $\Delta P>0 \mathrm{~Pa}$, except for the case of $\Delta P=0 \mathrm{~Pa}$ in Fig. 5, which is conducted in the wider part of the microchannel, not near the contraction. This is to elucidate the relation between the laser power and the temperature increase without disturbance by the presence of the contraction and flow field. In Fig. 5(a), experimental data are shown by two sets of symbols. Linear fits to the maximum temperature $T_{\max }$ are obtained by the leastsquares method up to a laser power of $100 \mathrm{~mW}$. It is clear that the maximum temperature $T_{\max }$ is proportional to the 


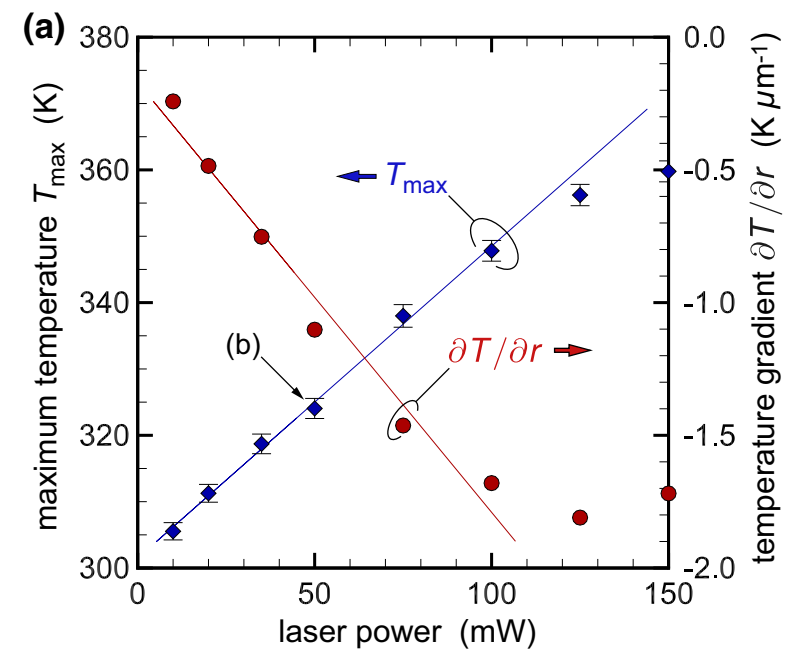

(b)

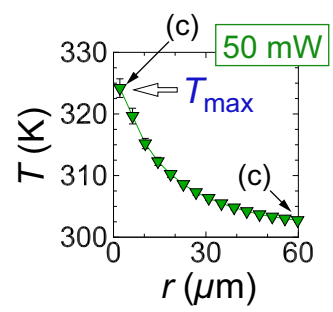

(c)

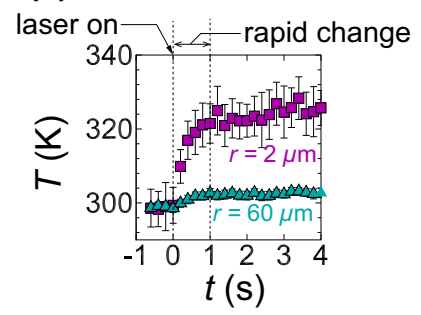

FIG. 5. (a) Maximum temperature $T_{\max }$ and radial temperature gradient $\partial T / \partial r$ induced by laser irradiation. The value of $\partial T / \partial r$ is evaluated at $r=r_{\max }$, where $|\partial T / \partial r|$ is maximum. Experimental data are shown by symbols. Linear fits to the experimental data are obtained by the least-squares method up to a laser power of $100 \mathrm{~mW}$. (b) Radial temperature profile for a laser power of $50 \mathrm{~mW}$. (c) Temporal development of the temperature at $r=2 \mu \mathrm{m}$ and $r=60 \mu \mathrm{m}$.

laser power if laser power is less than $100 \mathrm{~mW}$. The same can be said about the temperature gradient $\partial T / \partial r$ shown in the same figure. We expect that the radius $r_{\text {laser }}$ of the laser spot is similar to $r_{\max }$ and we thus conclude that $r_{\text {laser }} \approx 7.7 \mu \mathrm{m}$. The results for a laser power larger than $100 \mathrm{~mW}$, shown in Fig. 5(a), slightly deviate from the linear fits. This deviation is attributed to heat dissipating to the glass substrate and PDMS channel wall. In fact, when we use a laser power higher than $150 \mathrm{~mW}$, we observe deformation of the PDMS after several minutes of laser irradiation. The deformation is suspected to be caused by heat and not by the laser itself, because the deformation disappears a couple of hours after we turn the laser off.

Figure 5(b) shows the temperature profile for a laser power of $50 \mathrm{~mW}$. It is confirmed that the temperature is maximum at the laser irradiation point at $r=0 \mu \mathrm{m}$. Figure 5(c) presents the time development of the temperature at $r=2 \mu \mathrm{m}$ and $r=60 \mu \mathrm{m}$ in Fig. 5(b). For $r=2 \mu \mathrm{m}$, the error bars are relatively large because fewer pixels are used in the averaging process for smaller $r$. It is seen that the temperature rapidly increases within $1 \mathrm{~s}$ after the onset of laser irradiation. This time scale of the temperature increase is the same as that in our previous study [39], which was carried out in microchannels with similar dimensions.

Next, we present the temperature fields when flow is induced by the nonzero pressure difference. The laser is incident upon the entrance of the contraction, that is, the origin in Fig. 3(a). Temperature $T$ is obtained as the function of $r$ by analyzing the temperature profile in $x<0 \mu \mathrm{m}$. Figure 6 shows the temperature fields for typical parameter sets. Solid curves are the fitting to a Lorentzian curve [79,80,83]: $T(r)=T_{1} /\left[1+(r / \sigma)^{2}\right]+T_{0}$, where $T_{1}$ is the temperature increase at the origin, $T_{0}$ is the temperature at infinity, and $\sigma$ is the half-width at the midheight value of the temperature profile. It is seen that the fitting curves approximate the experimental data well. A comparison between Figs. 6(a) and 6(b), where the laser powers are 50 and $100 \mathrm{~mW}$, respectively, shows that the overall temperature profile is enhanced in Fig. 6(b) with higher laser power. The same is true for the comparison between Figs. 6(c) and 6(d). However, the comparison between Figs. 6(a) and 6(c) or between Figs. 6(b) and 6(d), where $\Delta P=0.5$ and $1.0 \mathrm{~Pa}$, respectively, indicates that the pressure difference $\Delta P$ yields a minor difference in the overall temperature field. It is remarkable that the magnitude of the temperature gradient for these cases can be greater than $1 \mathrm{~K} \mu \mathrm{m}^{-1}$. Such a large temperature gradient is observed in the manipulation of nanomaterials using a plasmonic effect [40,84-87], where thermophoretic effects, near-field optical effects, and convections are considered to coexist. Our approach, in contrast, tries to simplify the situation by focusing only on thermophoresis.

\section{B. Observation of laser-induced thermophoresis}

A mixed solution of PS beads (F8823) and silica beads $\left(d=1 \mu \mathrm{m}, 40-02-103\right.$, sicastar ${ }^{\circledR}$-redF, Micromod Partikeltechnologie, Rostock, Germany) is prepared. These two types of particles have different fluorescence characteristics, so we can choose which one to observe by using the appropriate optical setup. In the following, we basically observe only the PS particles using the U-FBW mirror unit. However, silica particles are also used and discussed in Sec. III C. The particle concentration is diluted to $0.1 \mathrm{wt} \%$ using a tris- $\mathrm{HCl}$ solution of $10 \mathrm{mmol}^{-1}$.

We show a typical result obtained using device $\mathrm{C}$ in Fig. 7, where $\Delta P=1.0 \mathrm{~Pa}$ and the laser power is 100 $\mathrm{mW}$. First, we confirm that the flow is absent, that is, $\Delta P=0 \mathrm{~Pa}$, by observing the motion of particles. We then apply the pressure difference $\Delta P>0 \mathrm{~Pa}$ to induce a flow in the microchannel. The laser is turned on at $t=0 \mathrm{~s}$ to irradiate the origin indicated in Fig. 1(a). The experimental protocol comprises three stages, as shown in Fig. 7(a): 

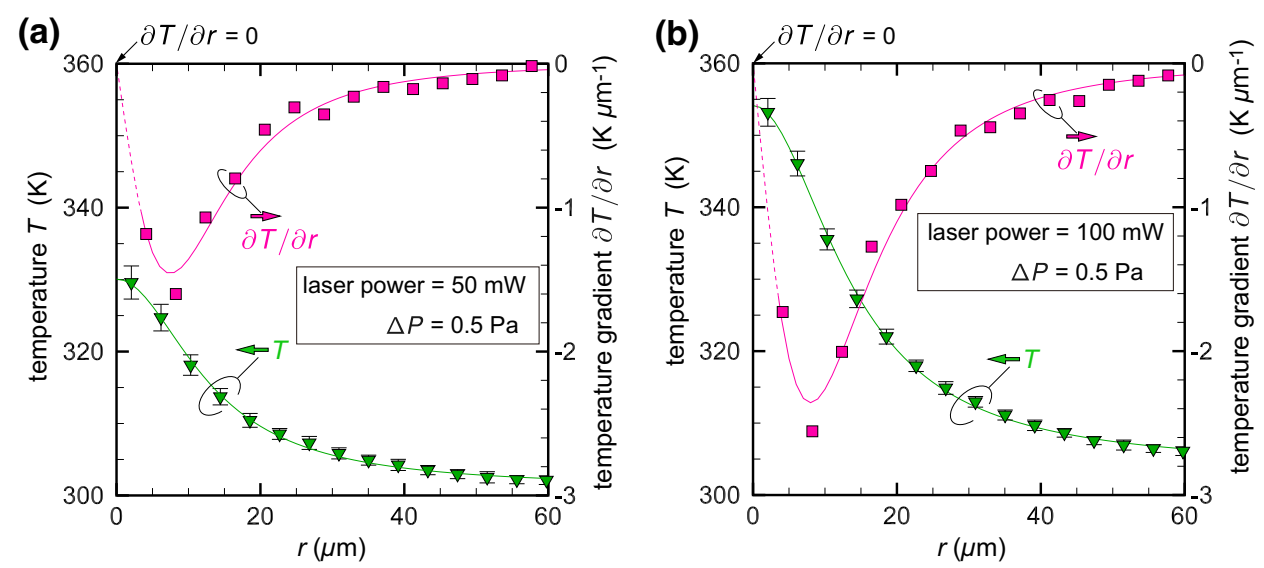

FIG. 6. Temperature field $T$ and temperature gradient $\partial T / \partial r$ as a function of $r$. (a) $\Delta P=0.5 \mathrm{~Pa}$ with a laser power of $50 \mathrm{~mW}$. (b) $\Delta P=0.5 \mathrm{~Pa}$ with a laser power of $100 \mathrm{~mW}$. (c) $\Delta P=1.0 \mathrm{~Pa}$ with a laser power of $50 \mathrm{~mW}$. (d) $\Delta P=1.0 \mathrm{~Pa}$ with a laser power of $100 \mathrm{~mW}$. Experimental data shown by symbols are well fitted by Lorentzian curves [79,80, 83]. Note that $\partial T / \partial r=0$ holds at $r=0$ due to the radial symmetry.


(i) particle translocation by the fluid flow resulting from a pressure difference $\Delta P=1 \mathrm{~Pa}$ at $t<0 \mathrm{~s}$;

(ii) obstruction of particle translocation by laserinduced thermophoresis for $0 \leq t \leq 180 \mathrm{~s}$, as shown in Fig. 7(b); and

(iii) restart of particle translocation for $t>180 \mathrm{~s}$, as shown in Fig. 7(c).

It is seen from Fig. 7(b), which shows snapshots during stage (ii), that the thermophoretic force acting on the particles is in the direction opposite to the temperature gradient and, thus, the particles are repelled from the heated region, which is the laser-irradiated position at the origin. During the laser irradiation, some particles in the ringlike pattern seem to form clusters and/or stick to the microchannel walls. However, after we turn the laser off, the particle translocation restarts through the contraction without clustering or sticking to the microchannel wall as shown in Fig. 7(c).

The results obtained with the same protocol for various $\Delta P$ and different device types are summarized in Fig. 8. We first focus on Figs. 8(a)-8(c), where the results of device $\mathrm{C}$ for $\Delta P=0.5,1.0$, and $1.5 \mathrm{~Pa}$ are presented. The upper image in each panel is a snapshot at $t=180 \mathrm{~s}$. Lower images are the relative fluorescence distributions with respect to time $t$ and radius $r$, which are obtained by projecting snapshots during $0 \leq t \leq 180 \mathrm{~s}$ onto the $t-r$ plane. For $\Delta P=0.5 \mathrm{~Pa}$ shown in Fig. 8(a), a ring pattern with a radius of $58 \mu \mathrm{m}$ is observed, where a thermophoretic repelling force from the origin in the negative $r$ direction counterbalances the flow drag in the positive $r$ direction (see Fig. 3). The dynamic ring formation can also be observed in Fig. 8(b). The formation of the ring is the consequence of increased particle concentration at this position. We denote $r_{\text {peak }}$ as the ring radius. For the larger pressure difference $\Delta P=1.0 \mathrm{~Pa}$ shown in Fig. $8(\mathrm{~b})$, the ring radius shrinks to $r_{\text {peak }}=27 \mu \mathrm{m}$. The result of the further pressure increase presented in Fig. 8(c) yields a ring of radius $r_{\text {peak }}=12 \mu \mathrm{m}$. Next, the results for device B are described, as shown in Figs. 8(d)-8(f). The pressure differences $\Delta P$ are set to those presented in Table II. For $\Delta P=0.1$ and $0.2 \mathrm{~Pa}$, rings with radii of $r_{\text {peak }}=68 \mu \mathrm{m}$ and $r_{\text {peak }}=25 \mu \mathrm{m}$ are observed, respectively. For $\Delta P=$ $0.5 \mathrm{~Pa}$, the ring shape is collapsed, but particle translocation is still obstructed. In such a situation, the optical force may have an effect near $r \approx 0 \mu \mathrm{m}$. A similar result is obtained for device $\mathrm{A}$, the results of which are presented in Figs. 8(g)-8(i) with $\Delta P$ as given in Table II. For device $\mathrm{A}$, ring collapse is observed with $\Delta P \geq 0.2 \mathrm{~Pa}$. It should be noted that in Figs. 8(f) and 8(i), where the rings are collapsed, many particles translocate the contraction without obstruction by the laser. Therefore, more precise control of $\Delta P$ in devices $\mathrm{A}$ and $\mathrm{B}$ is necessary to regulate the particle flow, while device $\mathrm{C}$ is less sensitive to small 




(b)
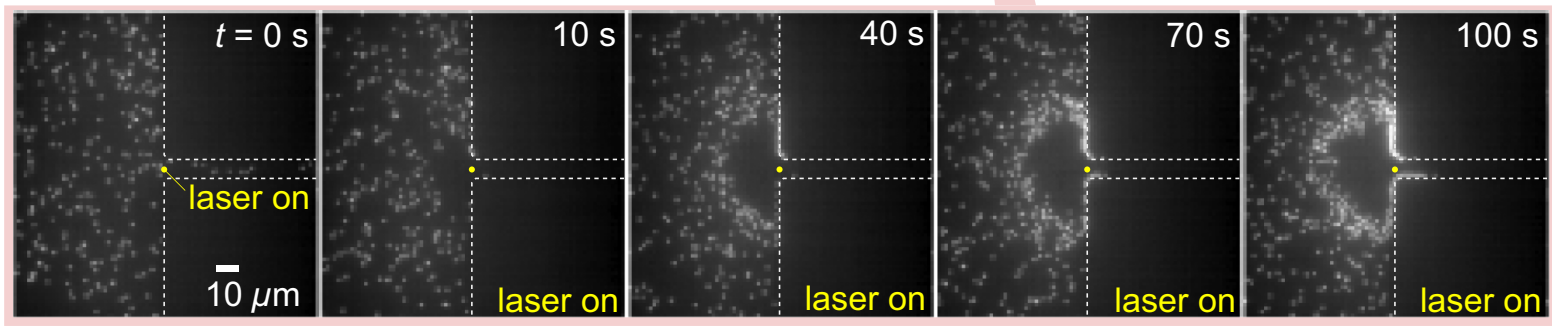

(c)
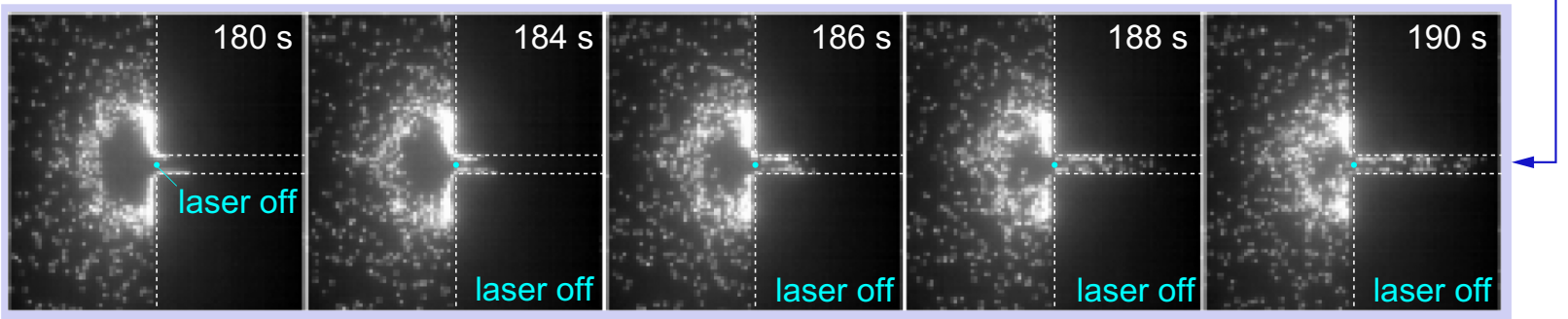

FIG. 7. (a) Thermophoretic obstruction of particle translocation into and through a contraction. Experimental protocol comprising three stages: (i) particle translocation by the fluid flow resulting from pressure difference $\Delta P=1 \mathrm{~Pa}$ at $t<0 \mathrm{~s}$; (ii) obstruction of particle translocation by laser-induced thermophoresis at $0 \leq t \leq 180 \mathrm{~s}$, shown in panel (b) (laser on at $t=0 \mathrm{~s}$ ); and (iii) restart of particle translocation for $t>180 \mathrm{~s}$, shown in panel (c) (laser off at $t=180 \mathrm{~s}$ ). Thermophoretic force acts upon particles in the direction opposite to the temperature gradient and thus the particles are repelled from the heated part, that is, the laser-irradiated position at the origin.

pressure changes and is thus preferable for obstructing particle translocation by thermophoresis.

We then carry out the same experiment using the smaller PS particles with a diameter of $d=100 \mathrm{~nm}(d=$ $0.099 \pm 0.008 \mathrm{~nm}, \mathrm{~F} 8803$, Molecular Probes, Eugene, USA) with a concentration of $10^{-2} \mathrm{wt} \%$ in device C. The results are shown in Fig. 9. Note that the only difference between Figs. 8(a)-8(c) and Figs. 9(a)-9(c) is the particle diameters $d$. The ring patterns are again observed as in Figs. 8(a)-8(c). However, the rings in Fig. 9 are less distinct due to the effect of diffusion. It should be noted that, in Figs. 9(b) and 9(c), some PS particles enter the contraction. These results indicate that, when external forces such as pressure difference are properly controlled, the present concept of particle control by laser-induced thermophoresis in the microchannel is effective not only for microparticles but also for nanoparticles. Thus, the results with the nanoparticles in Fig. 9 enlarge the range of applicability of the present concept to biomedical devices targeting biomolecules or viruses that have typical sizes of hundreds of nanometers [88]. For instance, the viruses of influenza are spherical or filamentous in shape with an equivalent diameter of $100 \mathrm{~nm}$ [89]; those of HIV-1 are approximately spherical with an equivalent diameter of $125 \mathrm{~nm}$ [90]; and Mimivirus, which is known as a large virus, has a particle size of $750 \mathrm{~nm}$ [91]. One of the authors, along with colleagues, developed a nanofluidic device for the detection of pollen allergen particles, which has a diameter of $330 \mathrm{~nm}$ on average [5]. Therefore, the sizes of these viruses and allergen particles are contained in the range investigated in this paper and the detection of these biological nano-objects is the scope of the application. The thermophoretic properties of the above samples are still unknown and should be investigated in future work. In contrast, the thermophoretic characteristics of DNA molecules [16,18,19,21,75,83] and proteins [20,42,43] have been investigated and, thus, such biomolecules are expected to be controllable with the method proposed in 


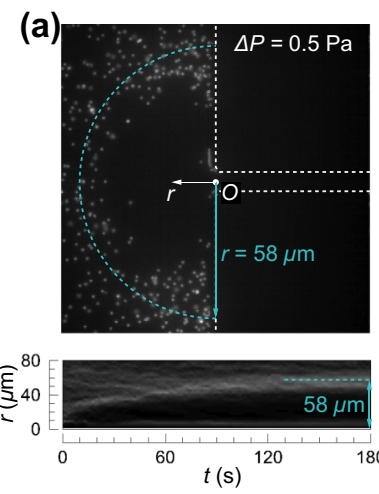

(b)

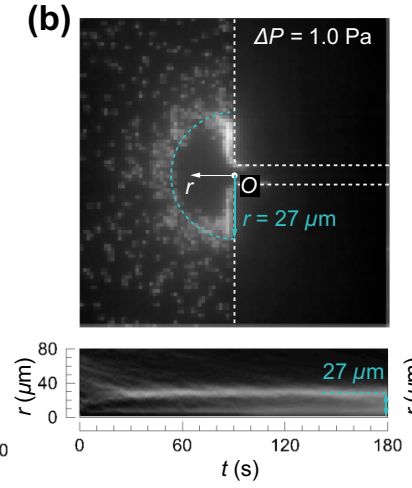

(c)
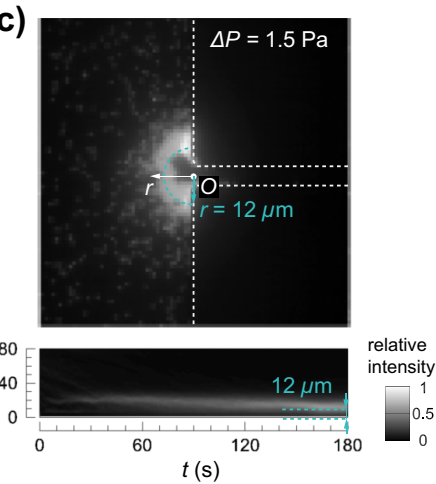

(d)

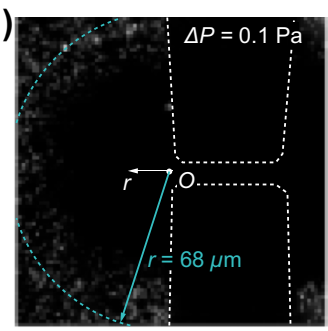

(e)

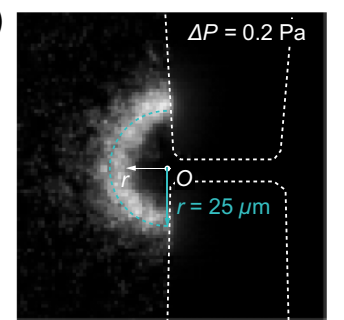

(f)
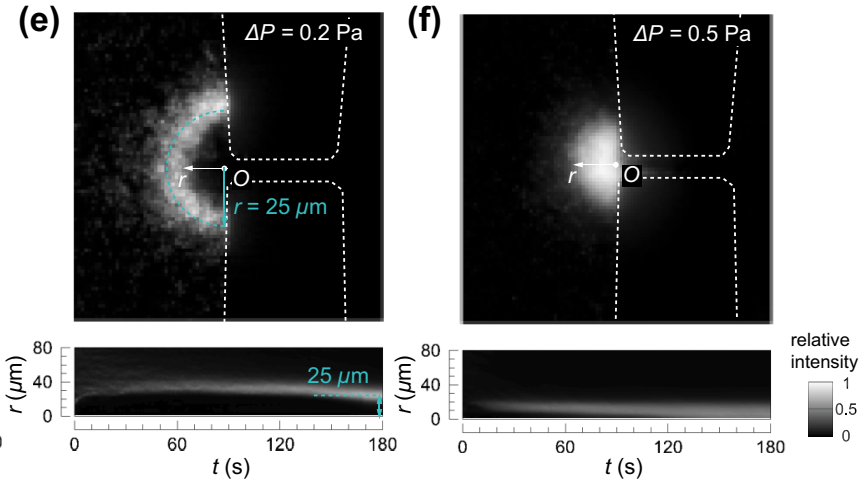

(i)

(h)
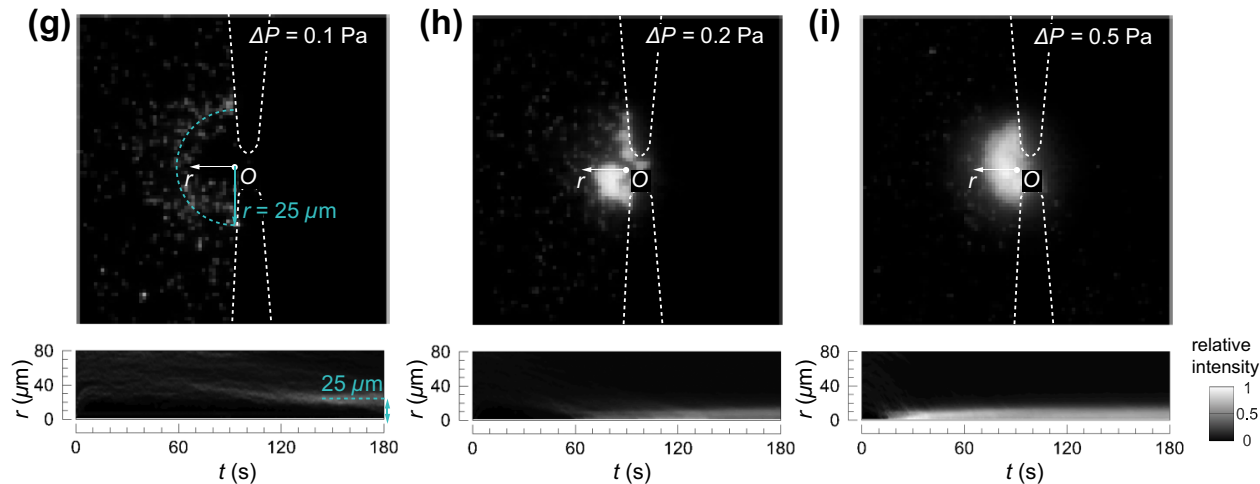

FIG. 8. Results of thermophoretic obstruction for various pressure differences $\Delta P$ in (a)-(c) device C, (d)-(f) device $\mathrm{B}$, and (g)-(i) device $\mathrm{A}$. The particle diameter is $d=1 \mu \mathrm{m}$. Upper images in each panel are snapshots at $t=180 \mathrm{~s}$ and lower images are the relative fluorescence distributions with respect to time $t$ and radius $r$. Ring patterns are observed in panels (a)-(e) and $(\mathrm{g})$, where a thermophoretic repelling force in the negative $r$ direction counterbalances flow drag in the positive $r$ direction. this paper. However, the large temperature rise needed to induce thermophoresis can be a problem for some biological samples. Since the magnitude of thermophoresis is proportional to the temperature gradient, a further downsizing of the heat source is expected to solve this problem, by decreasing the temperature rise while maintaining the magnitude of the temperature gradient. Such a downsizing may be achieved by using an objective lens with a higher NA value to reduce the size of the focal spot and/or by using plasmonic nanostructures to localize the optical effect [40,84-87].

We estimate the thermophoretic mobility $D_{T}$ $\left(\mu \mathrm{m}^{2} \mathrm{~s}^{-1} \mathrm{~K}^{-1}\right)$ from the above experiment with device C. Under the temperature gradient $\nabla T$, the thermophoretic mobility is defined as $\mathbf{v}_{T}=-D_{T} \nabla T$, where $\mathbf{v}_{T}$ is the thermophoretic velocity of the particle $[13,15]$. As discussed in Sec. III 2, the temperature field can be considered as the function of $r$ for $x<0$; therefore, $\mathbf{v}_{T}$ has only the $r$-component, which is denoted by $v_{T, r}(r)=-D_{T} \frac{\partial T}{\partial r}$. In contrast, as discussed in Sec. III 1, the flow velocity has only the $r$ component, $u_{r}$, for $x<0$ and $u_{r}$ is a function of $r$. Therefore, the particle velocity $\mathbf{v}$ has only the $r$-component, that is, $v_{r}=v_{r}(r)$. We assume that $v_{r}$ can be written as $v_{r}=u_{r}+v_{T, r}$. Therefore, by detecting the position $r_{0}$ such that $v_{r}=0 \mu \mathrm{m} \mathrm{s}^{-1}$, we can evaluate the thermophoretic mobility $D_{T}$ from the relation $D_{T}=\left.\left(u_{r} / \frac{\partial T}{\partial r}\right)\right|_{r=r_{0}}$. Because we have $v_{r}=0$ at position $r_{0}$, the particles are expected to accumulate at $r=r_{0}$, which should coincide with the $r=r_{\text {peak }}$ shown in Figs. 8 and 9. In summary, we obtain the values of $D_{T}$ by investigating the ring radius $r=r_{\text {peak }}$. Note that we ignore optical forces acting on the particle. As estimated in Appendix B, 


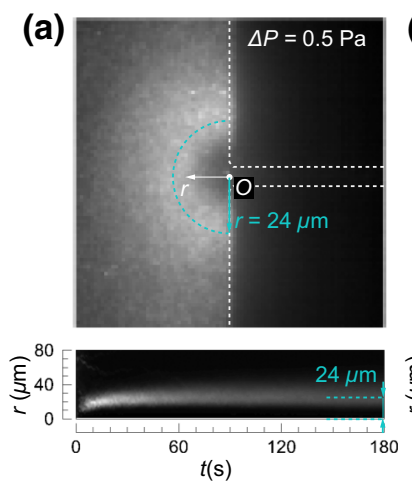

(b)


FIG. 9. Results of thermophoretic obstruction for various pressure differences $\Delta P$ in device $\mathrm{C}$ with particle diameter of $d=100 \mathrm{~nm}$. The ring patterns observed in Figs. 8(a)-8(c) are less distinct due to diffusion. the optical forces are less than $1 \mathrm{fN}$ for $r>12.5 \mu \mathrm{m}$, while the drag and thermophoretic forces are $O(10) \mathrm{fN}$. In fact, the magnitude of the thermophoretic force can be estimated from the experimental result as follows. Thermophoretic force is counterbalanced by the Stokes drag $6 \pi \eta(d / 2) v_{T, r}$, where $\eta=8.5 \times 10^{-4} \mathrm{~Pa}$ s is the viscosity of the solution at $300 \mathrm{~K}$. Using the relation $v_{T, r}(r)=-D_{T} \frac{\partial T}{\partial r}$ with $D_{T}$ and $\left.\frac{\partial T}{\partial r}\right|_{r=r_{\text {peak }}}$ given by Table III and Fig. 6, respectively, we can calculate the magnitude of thermophoretic force as $7.4 \mathrm{fN}$ at $313 \mathrm{~K}$ for $\Delta P=1.0 \mathrm{~Pa}$ and laser power of $100 \mathrm{~mW}$.

Table III shows $D_{T}$ for various laser powers, pressure differences $\Delta P$, and particle diameters $d$. First, let us focus on the case with $d=1 \mu \mathrm{m}$. It is seen that $D_{T}$ is within the range $0.9 \leq D_{T} \leq 3.2 \mu \mathrm{m}^{2} \mathrm{~s}^{-1} \mathrm{~K}^{-1}$, which is of the same order of magnitude as in other studies (e.g., $[13,16,28,29,39])$. However, $D_{T}$ tends to be smaller for larger $\Delta P$, which needs some explanation. In the case of $d=1 \mu \mathrm{m}$, the particles have a diameter smaller than the channel height, but the channel height may not be high enough to exclude the volume effect of the particle. To be more precise, the particles in the ringed distribution may pile up in the microchannel. This pileup shrinks the

TABLE III. Thermophoretic mobility $D_{T}$ obtained using device $\mathrm{C}$ for various conditions. The values in parentheses are the results obtained with the diluted particle dispersion.

\begin{tabular}{lcccc}
\hline \hline $\begin{array}{l}\text { Laser } \\
\text { power } \\
(\mathrm{mW})\end{array}$ & $\begin{array}{c}\Delta P \\
(\mathrm{~Pa})\end{array}$ & $\begin{array}{c}r_{\text {peak }}(\mu \mathrm{m}) \\
\left(\mu \mathrm{m}^{2} \mathrm{~s}^{-1} \mathrm{~K}^{-1}\right)\end{array}$ & $d(\mu \mathrm{m})$ \\
\hline 50 & 0.5 & $32(34)$ & $2.34(2.56)$ & 1 \\
50 & 1.0 & $9(23)$ & $1.97(2.48)$ & 1 \\
50 & 1.5 & $\left(r_{\text {peak }}\right.$ & is smaller than $\left.r_{\text {laser }}\right)$ & 1 \\
100 & 0.5 & $58(64)$ & $3.21(3.85)$ & 1 \\
100 & 1.0 & $27(40)$ & $1.49(2.57)$ & 1 \\
100 & 1.5 & $12(29)$ & $1.02(1.63)$ & 1 \\
150 & 0.5 & $\left(r_{\text {peak }}\right.$ is out of observation range $)$ & 1 \\
150 & 1.0 & $42(48)$ & $1.81(2.22)$ & 1 \\
150 & 1.5 & $24(38)$ & $0.94(1.62)$ & 1 \\
100 & 0.5 & 24 & 0.87 & 0.1 \\
100 & 1.0 & 17 & 1.04 & 0.1 \\
100 & 1.5 & 11 & 1.05 & 0.1 \\
\hline \hline
\end{tabular}

effective channel height, and leads to increased flow speed because of the conservation of mass. The pileup effect must be enhanced for higher $\Delta P$ and smaller $r_{\text {peak }}$, since the particle density increases faster under such conditions. Therefore, a larger $\Delta P$ results in smaller $D_{T}$, as shown in Table III. To check the effect of the concentration of particle dispersion, we carry out the same experiment using a 20-fold diluted solution. The results are summarized in Table III in parentheses. It is seen that measured $D_{T}$ slightly increases to $1.6 \leq D_{T} \leq 3.9 \mu \mathrm{m}^{2} \mathrm{~s}^{-1} \mathrm{~K}^{-1}$, and the range of variation becomes smaller. Since the results with a weak laser power of $50 \mathrm{~mW}$ show similar values of $D_{T}$ for different $\Delta P$, the use of a weak laser power to create a larger ring radius (equivalently, a smaller pileup effect) may be effective for more accurate measurement of $D_{T}$. Such a speculation on pileup effect is further supported by the results for the smaller particle of $d=0.1 \mu \mathrm{m}$ presented in Table III. In contrast to the case of $d=1 \mu \mathrm{m}, D_{T}$ is almost constant with respect to $\Delta P$ in the case of $d=$ $0.1 \mu \mathrm{m}$, although $D_{T}$ slightly increases as $\Delta P$ increases. Since the volume of the particle with $d=0.1 \mu \mathrm{m}$ is very much smaller than that with $d=1 \mu \mathrm{m}$, the volume effect mentioned earlier is negligible for the case of $d=0.1 \mu \mathrm{m}$. The slight increase of $D_{T}$ is caused by the fact that $D_{T}$ usually tends to be larger as $T$ increases [28], which happens as $r$ becomes smaller (Fig. 6) in this study.

It should be noted that a temperature gradient smaller than that used in this study is usually used for the investigation of the value of $D_{T}$ to avoid complexity. Here, the temperature gradient is chosen to be large to counterbalance the Stokes drag and thermophoretic force. Although we consider that $D_{T}$ is independent of the magnitude of the temperature gradient, as experimentally shown in, for example, Ref. [34], the effect of the large temperature gradient should be further investigated in future work.

\section{Control of particle translocation through contraction}

This section characterizes and demonstrates how the present method controls particle translocation into and through the contraction. 
(a)

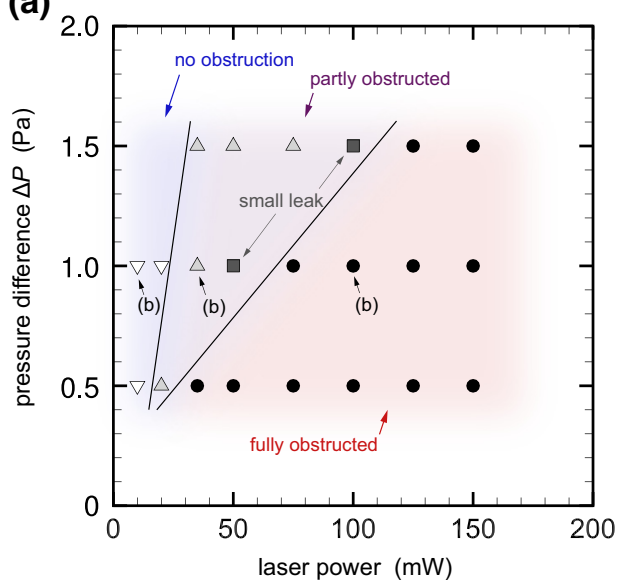

(b)

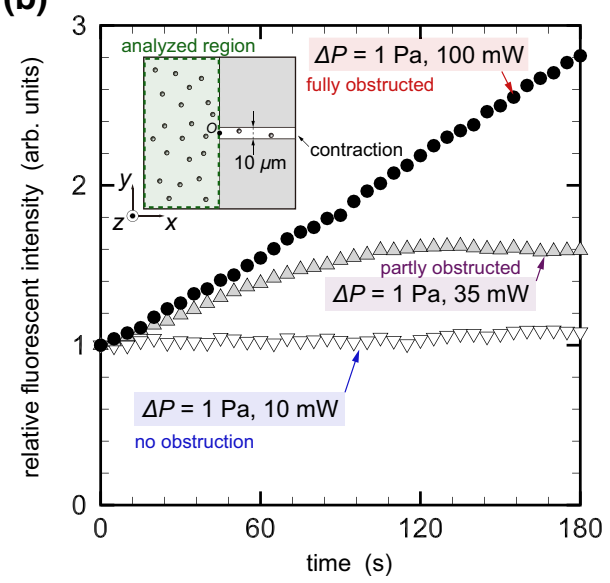

FIG. 10. (a) Characterization of thermophoretic obstruction of particle translocation into the contraction within a parameter space of laser power and pressure difference $\Delta P$. Inverted triangles denote that no obstruction is observed; triangles, particle translocation is partly obstructed; squares, particle translocation is obstructed, but a small leak is observed; and circles, particle translocation is fully obstructed. (b) Temporal development of relative fluorescence intensity for the region $x<0$ is indicated in the inset. The fluorescence intensity is normalized by the intensity at $t=0 \mathrm{~s} . \Delta P=1 \mathrm{~Pa}$ and the laser power is 10 (no obstruction), 35 (partly obstructed), and 100 $\mathrm{mW}$ (fully obstructed). At $10 \mathrm{~mW}$, no change in the intensity is observed. At $35 \mathrm{~mW}$, the intensity increases with time but saturates, where the number of particles coming into and going out of the analyzed region is equal. The intensity increases with a constant rate at $100 \mathrm{~mW}$, which indicates the complete obstruction of the particle translocation into the contraction.

\section{Characterization of thermophoretic obstruction}

Figure 10(a) shows a schematic classification of thermophoretic obstruction obtained by a systematic investigation of the laser power and the pressure difference. At low laser power $(<30 \mathrm{~mW})$, no obstruction of particle translocation is observed, as indicated by inverted triangles in the figure. As the laser power increases, particle translocation is partly obstructed by laser-induced thermophoresis, but leakage into the contraction is still observed. This condition is indicated by grey triangles in Fig. 10(a), and we use squares to mark the cases with small leakage. When the laser power is high and/or the pressure difference is small, particle translocation into the contraction is fully obstructed. Figure 10(b) shows the temporal development of relative fluorescence intensity in the region $x<0$ indicated in the inset. The fluorescence intensity is normalized by that at $t=0 \mathrm{~s}$. $\Delta P$ is set to $1 \mathrm{~Pa}$, and the laser power is 10 (no obstruction), 35 (partly obstructed), and $100 \mathrm{~mW}$ (fully obstructed). At $10 \mathrm{~mW}$, no change in the fluorescence intensity is observed; that is, no obstruction occurs. In contrast, at $35 \mathrm{~mW}$, the fluorescence intensity gradually increases over time. However,
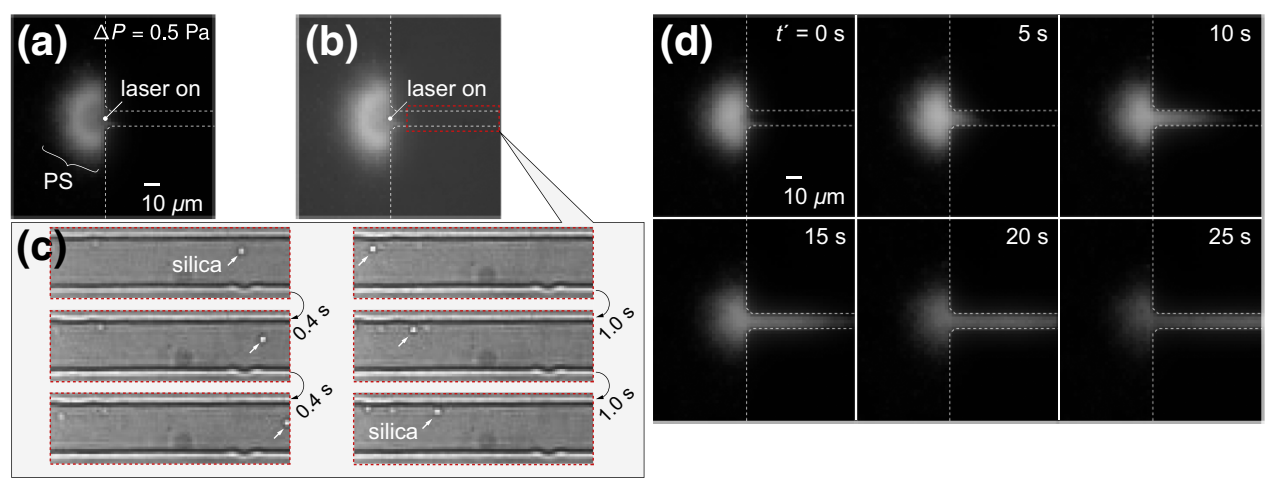

FIG. 11. Schematics of selective particle translocation with $\Delta P=0.5 \mathrm{~Pa}$. (a) Dark-field observation of PS particles, translocation of which is obstructed by laser-induced thermophoresis. (b) Bright-field observation of the same experiment, with translocation of nonfluorescent silica particles into the contraction, which is magnified in panel (c). (c) Two examples of selective silica-particle translocation. To enhance the visibility of the silica particles, the contrast of each snapshot is adjusted using image-editing software (Image J 1.50a, National Institutes of Health, USA). (d) Dark-field observation of PS particles after the laser is turned off at $t^{\prime}=0 \mathrm{~s}$ and they are flushed out. 




FIG. 12. Schematic and results of the proposed translocation control technique. (a) Translocation speed $\bar{u}_{x}$ and number of translocating particles per unit time $f_{\text {detect }}$ are evaluated for three cases: case 1 , the pressure difference $\Delta P$ is 0.3 Pa without laser irradiation; case 2, $\Delta P$ is $1.0 \mathrm{~Pa}$ without laser irradiation; and case $3, \Delta P$ is $1.0 \mathrm{~Pa}$ for $t<250 \mathrm{~s}$ and $\Delta P=0.3 \mathrm{~Pa}$ for $t>250 \mathrm{~s}$ with laser irradiation for $10<t<250 \mathrm{~s}$. (b) Schematic of case 3. Case 3 consists of two phases: an accumulation phase to increase the number of particles near the contraction entrance and a detection phase to transport the particles. (c) Experimental results. The black, blue, and red curves represent the results of cases 1,2, and 3, respectively, showing the relative fluorescence intensity as a function of time. The intensity is measured at specific sensing locations with an area of $0.51 \times 0.51 \mu \mathrm{m}^{2}$ and the spikes indicate particle translocation at the sensing locations. In case $1, \bar{u}_{x}=1.5 \mu \mathrm{m} \mathrm{s}^{-1}$ is obtained, which is smaller than $\bar{u}_{x}=5.3 \mu \mathrm{m} \mathrm{s}^{-1}$ in case 2 , and, thus, case 1 is better with regard to detection accuracy. However, $f_{\text {detect }}=3.4$ counts $\min ^{-1}$ is obtained in case 1 , which is lower than $f_{\text {detect }}=12.3$ counts min ${ }^{-1}$ in case 2 . Hence, case 2 has better detection frequency. Case 3 results in a translocation speed of $\bar{u}_{x}=2.4 \mu \mathrm{m} \mathrm{s}{ }^{-1}$, which is smaller than that of case 2 , and a detection frequency of 10.5 counts $\mathrm{min}^{-1}$, which is larger than that of case 1 . Since the experiment duration is the same for all cases, case 3 is the best for increasing the detection frequency and reducing the translocation speed, achieving better overall performance for use in a sensor.

the intensity saturates at a certain value, where the number of particles entering and leaving the analyzed region is equal. At $100 \mathrm{~mW}$, the fluorescence intensity increases at a constant rate. This increasing intensity behavior indicates the full obstruction of particle translocation into the contraction. The present characterization result can be used as a first step to establish the optimal design of the contraction for broader application in biomedical devices.

\section{Selective particle translocation}

The mixed solution of PS and silica particles explained in Sec. III B is used to demonstrate selective particle translocation into the contraction. Note that the PS particles are thermophobic; that is, they are repelled by the high-temperature region, while silica particles are not [39].

First, we pile up the PS particles using the flow field induced by $\Delta P=0.5-1.0 \mathrm{~Pa}$. The laser power is $75 \mathrm{~mW}$. More than $15 \mathrm{~min}$ after the laser irradiation, a pileup of PS particles is observed, as shown in Fig. 11(a). Note that only the PS particles can be seen due to our choice of the mirror unit. We then use the transmitted light to observe the silica particles [Fig. 11(b)]. Figure 11(c) presents magnified views of the contraction, where translocation of nonfluorescent silica particles is observed. That is, the PS particles are repelled from the contraction entrance, while the silica particles are drawn to it. Figure 11(d) again shows the dark-field view. The laser irradiation is halted at $t^{\prime}=0 \mathrm{~s}$ 
and the subsequent images of the contraction are presented for $t^{\prime}=5, \ldots, 25 \mathrm{~s}$. It is seen that, after we turn the laser off, the PS particles start to translocate into the contraction. The above demonstration shows that the proposed method using laser-induced thermophoresis near the entrance of the contraction can induce selective particle translocation.

\section{Tuning translocation velocity and frequency}

In this section, we assume that the flow speed is equal to the particle-translocation speed in the contraction. Therefore, the PIV results presented below can be interpreted as the particle translocation speed. We use device $\mathrm{C}$ for all the experiments described in this section.

Figure 12 is an overview of the experiment described in this section, which aims to control both the translocation speed and translocation frequency of particles in the contraction [see Fig. 12(a)]. It should be noted that we prefer a slow translocation speed $\bar{u}_{x}$. A slower translocation speed leads to higher resolution and accuracy of the detection and recognition of translocating objects, since the detection is based on the transient changes in ionic or tunneling current [60] during translocation. In contrast, we prefer a high translocation frequency $f_{\text {detect }}$ to carry out stochastic analysis of the detected signals [70]. Therefore, increas$\operatorname{ing} f_{\text {detect }}$ while slowing $\bar{u}_{x}$ is desired for better detection performance of objects translocating the contraction geometry, such as in nanopore sensors. However, on the one hand, increasing $f_{\text {detect }}$ by a strong driving force induces a fast translocation speed. On the other hand, decreasing the translocation speed with a weak driving force causes a low detection frequency $f_{\text {detect }}$. We propose a method to resolve this problem using the technique presented in this paper.

Three cases are tested as shown in Fig. 12:

Case 1: $\Delta P=0.3 \mathrm{~Pa}$ without laser irradiation,

Case 2: $\Delta P=1.0 \mathrm{~Pa}$ without laser irradiation, and

Case 3: $\Delta P=1.0 \mathrm{~Pa}$ with laser irradiation from $t=$ 10 to $250 \mathrm{~s}$ and $\Delta P$ is changed to $0.3 \mathrm{~Pa}$ at $t=250 \mathrm{~s}$ [Fig. 12(b)].

Cases 1 and 2 are the reference experiments to be compared with our proposed method in case 3 . Case 3 consists of two phases as shown in Fig. 12(b): an accumulation phase to increase the number of particles near the contraction entrance $(t<250 \mathrm{~s})$ by laser-induced thermophoresis and a detection phase to transport the particles $(t>250 \mathrm{~s})$.

We evaluate the performance of particle translocation control using the values of the translocation speed $\bar{u}_{x}$ and the translocation frequency $f_{\text {detect }}$ for these three cases. The translocation speed $\bar{u}_{x}$ is obtained by the PIV analysis for the entire contraction channel and the translocation frequency $f_{\text {detect }}$ is obtained as follows. We prepare 16 sensing locations having an area of $0.51 \times 0.51 \mu \mathrm{m}^{2}$ in the contraction channel. When we plot the relative fluorescence intensity at each sensing location as a function of time [see Fig. 12(c), which shows the results for one particular sensing location], it shows a spikelike signal when a particle passes through the sensing location. We summarize the number of spikelike signals for all sensing locations to obtain $f_{\text {detect }}$. The curves in Fig. 12(c) with black, blue, and red colors represent the results for cases 1, 2, and 3, respectively. A comparison between the results of cases 1 $(\Delta P=0.3 \mathrm{~Pa})$ and $2(\Delta P=1.0 \mathrm{~Pa})$ shows that the higher $\Delta P$ results in much more frequent particle translocation as a natural consequence. The results of case 3 show that no particle translocation occurs while the laser is on, but many particle translocations are observed shortly after the laser is turned off.

In case $1, \bar{u}_{x}=1.5 \mu \mathrm{m} \mathrm{s}^{-1}$ is obtained from the PIV results, which is smaller than $\bar{u}_{x}=5.3 \mu \mathrm{m} \mathrm{s}^{-1}$ obtained in case 2 , and, thus, case 1 has a better detection accuracy. In contrast, $f_{\text {detect }}=3.4$ counts $\mathrm{min}^{-1}$ is obtained in case 1 , which is less than $f_{\text {detect }}=12.3$ counts $\min ^{-1}$ obtained in case 2 . Hence, case 2 has better detection frequency. Case 3 results in a translocation speed $\bar{u}_{x}=2.4 \mu \mathrm{m} \mathrm{s}^{-1}$, which is smaller than that of case 2 , and a detection frequency of 10.5 counts $\mathrm{min}^{-1}$, which is larger than that of case 1 . Since the device and the experimental duration are same for all cases, case 3 is the best for increasing the detection frequency and reducing the translocation speed and it achieves better overall performance as a sensor.

\section{CONCLUDING REMARKS}

In this study, we systematically investigate the nearinfrared laser-induced thermophoresis of micro- and nanoparticles in microchannels and its effect on particle translocation into and through a sudden contraction. The outcomes are summarized as follows:

(1) The relation between laser-induced thermophoresis of PS particles and flow/temperature fields is investigated for various parameters, such as laser power, pressure difference, contraction geometry, and particle size.

(2) The obstruction of particle translocation by laserinduced thermophoresis is characterized and classified by observing the PS particle pattern formed near the contraction.

(3) Demonstrations of the proposed particle manipulation technique with laser-induced thermophoresis are presented, namely, to selectively translocate particles in a mixture of different particles and to control the particletranslocation velocity and frequency.

The present method can be implemented without additional difficult fabrications, so it can be integrated into existing microfluidic devices having sensor or filter functions. Moreover, the dynamical control of the number density of nanomaterials using both flow fields and 
laser-induced thermophoresis with rapid on/off switching may lead to the development of controls for sequential chemical reactions in the microchannel. Because the laser is near infrared, the method is applicable not only to transparent devices but also to substrates opaque to visible light, such as silicon. In particular, silicon has a negligible absorption of light with wavelengths from 1.2 to $4 \mu \mathrm{m}$, which includes the wavelength $1475 \mathrm{~nm}$ used in this study [92]; that is, the absorption coefficient is less than $3 \times 10^{-2} \mathrm{~m}^{-1}$ within this range of wavelengths $[92,93]$. Further investigations of the other important parameters, such as salt concentrations of a solvent and particle nature, will be the topics of future study to extend the range of applications to biomedical fluid devices.

\section{ACKNOWLEDGMENTS}

The present study is supported by the Japan Society for the Promotion of Science (JSPS) KAKENHI Grant No. JP15K17973 for Young Scientists (B), JSPS KAKENHI Grant No. 18K13687 for Young Scientists, JSPS KAKENHI Grant No. 18H05242 for Scientific Research (S), and JSPS KAKENHI Grant No. JP16H06504 in Scientific Research on Innovative Areas "Nano-Material OpticalManipulation.”

\section{APPENDIX A: ESTIMATION OF MEAN FLOW SPEED IN CONTRACTION}

We approximate the present microfluidic channel of height $H$ as follows. A contraction of width $W$ and length $L$ connects two wider channels of width $W_{1}$ and length $L_{1}$. The cross-sectional areas for these channels are denoted by $A=W H$ and $A_{1}=W_{1} H$, respectively. The solution in the microchannel is incompressible and Newtonian with a constant and uniform viscosity $\eta=8.5 \times 10^{-4} \mathrm{~Pa} \mathrm{~s}$ at $300 \mathrm{~K}$. For the flow velocity $\bar{u}_{x}=O(10) \mu \mathrm{m} \mathrm{s}^{-1}$ in the contraction, the Re based on the channel length is $\operatorname{Re}=\rho L \bar{u}_{x} / \eta<3 \times 10^{-3}$. In other words, the flows in this study are assumed to be flows with a low Re number. We then assume that the flow is steady and fully laminar through the entire channel. The steady-state approximation is legitimized by the fact that the reservoirs are syringes with a cross-sectional area $A_{\text {res }}$ of $2 \times 10^{2} \mathrm{~mm}^{2}$, which is much larger than the cross-sectional area $A$ of the contraction. In our study, $A$ is evaluated as $A<10^{-4} \mathrm{~mm}^{2}$ (approximately $5 \times 10^{-7} \times A_{\text {res }}$ ). In other words, the pressure difference $\Delta P$ can be assumed to be constant during the experiment. Note that the experiment for a time duration $t_{0}$, for example, $5 \mathrm{~min} t_{0}=3 \times 10^{2} \mathrm{~s}$, results in a change in a water-level difference $\Delta h_{0}=\left(\bar{u}_{x} A t_{0}\right) / A_{\text {res }} \approx$ $1.5 \times 10^{-3} \mu \mathrm{m}$, which is negligibly small compared with the resolution of the control of $\Delta h$, that is, $\Delta h_{\min }=1 \mu \mathrm{m}$.

The pressure difference $\Delta P$ between the inlet and outlet is compensated by pressure losses through the microchannel. Using Bernoulli's theorem, we can associate the pressure losses with the mean flow velocity. In this study, we ignore minor losses such as velocity head or entrance and enlargement losses and we take only friction losses into account as the pressure loss. The friction losses are proportional to the hydraulic resistance, which can be explicitly obtained for channels with rectangular cross sections [81]. We let $R_{1}$ and $R$ denote the hydraulic resistances for the wider channels and contraction, respectively, and $\bar{u}_{1, x}$ and $\bar{u}_{x}$ denote the mean flow speed in the $x$ direction for the corresponding channels. Then, Bernoulli's theorem is analogous to Ohm's law [76,77] to find $\Delta P=R_{1} A_{1} \bar{u}_{1, x}+$ $R A \bar{u}_{x}+R_{1} A_{1} \bar{u}_{1, x}$, where the first and third terms in the right-hand side are the friction losses in the wider channels and the second term is friction loss in the contraction. Note that the equation of continuity leads to $A_{1} \bar{u}_{1, x}=A \bar{u}_{x}$. These equations are solved to obtain the relation $\bar{u}_{x}=\alpha \Delta P$ used in the main text.

\section{APPENDIX B: DESCRIPTION OF LASER AND OPTICAL FORCES ACTING ON PARTICLES}

The laser in this study is a Gaussian beam [94]. The laser intensity $I\left(\mathrm{~W} \mathrm{~m}^{-2}\right)$ is defined as

$$
I(r, z)=\frac{2 P_{\text {laser }}}{\pi w^{2}(z)} \exp \left[-\frac{2 r^{2}}{w^{2}(z)}\right]
$$

where $P_{\text {laser }}(\mathrm{W})$ is the laser power incident on the sample, $w(z)=w_{0}\left[1+\left(z-z_{0}\right)^{2} / \tilde{z}^{2}\right]^{1 / 2}$ is the beam waist, $w_{0}$ is the beam waist at the focal plane $z=z_{0}, \tilde{z}=\pi w_{0}^{2} n_{f} / \lambda$ is half of the depth of focus [94], and $n_{f}=1.33$ is the refractive index of the solution. In our experiments, we have $\lambda=1475 \mathrm{~nm}, z_{0}=0 \mu \mathrm{m}$ at the bottom of the microchannel, and $w_{0}=7.7 \mu \mathrm{m}$ as estimated from the experiments. Since the particles are confined in a narrow slit in the $z$ direction, we only consider an in-plane optical force acting on the particle in the radial direction $r$; that is, the optical force $\mathbf{F}_{\text {opt }}$ has only the $r$ component $F_{r \text {,opt }}$. In this study, the particle radius is smaller than that of the wavelength of the laser. Therefore, the optical force can be described by the Lorenz-Mie theory or Rayleigh approximation [95]. For a particle of diameter $\leq 1 \mu \mathrm{m}$ in this study, these two theories give quantitatively similar values for $F_{r \text {,opt }}$ [95]. Therefore, the optical forces acting on the particles are estimated as $F_{r \text {,opt }}=\left(\alpha_{\text {pol }} / 4\right) \nabla|\mathbf{E}|^{2}$ using Rayleigh approximation, where $\alpha_{\text {pol }}=4 \pi \epsilon_{f}(d / 2)^{3}\left(\epsilon_{p}-\right.$ $\left.\epsilon_{f}\right) /\left(\epsilon_{p}+2 \epsilon_{f}\right.$ ), $\epsilon_{f}$ (or $\epsilon_{p}$ ) is the permittivity of fluids (or particles), and $\mathbf{E}$ is the electric-field vector of the Gaussian beam propagating from the negative $z$ to the positive $z$. Therefore, $|\mathbf{E}|^{2}=2 /\left(n_{f} c \epsilon_{0}\right) I$, where $\epsilon_{0}$ is the permittivity in a vacuum and $c$ is the speed of light. Using the material properties $\epsilon_{p}=n_{p}^{2} \epsilon_{0}$ and $\epsilon_{f}=n_{f}^{2} \epsilon_{0}$ at $298 \mathrm{~K}$, where $n_{p}=1.57$ is the refractive index of the particle [96], we can compute the magnitude of $F_{r \text {,opt }}$ as $\left|F_{r, \text { opt }}\right|<1 \mathrm{fN}$ at 
$r=12.5 \mu \mathrm{m}$ and it rapidly decreases as $r$ increases. Therefore, when compared with the drag and thermophoretic forces, the optical force can be negligible for $r>12.5 \mu \mathrm{m}$, which is the region of main concern in this study.

[1] E. K. Sackmann, A. L. Fulton, and D. J. Beebe, The present and future role of microfluidics in biomedical research, Nature 507, 181 (2014).

[2] E. F. Hasselbrink, T. J. Shepodd, and J. E. Rehm, Highpressure microfluidic control in lab-on-a-chip devices using mobile polymer monoliths, Anal. Chem. 74, 4913 (2002).

[3] W. A. Braff, A. Pignier, and C. R. Buie, High sensitivity three-dimensional insulator-based dielectrophoresis, Lab Chip 12, 1327 (2012).

[4] R. Vogel, W. Anderson, J. Eldridge, B. Glossop, and G. Willmott, A variable pressure method for characterizing nanoparticle surface charge using pore sensors, Anal. Chem. 84, 3125 (2012).

[5] C. Kawaguchi, T. Noda, M. Tsutsui, M. Taniguchi, S. Kawano, and T. Kawai, Electrical detection of single pollen allergen particles using electrode-embedded microchannels, J. Phys.: Condens. Matter 24, 164202 (2012).

[6] N. Yukimoto, M. Tsutsui, Y. He, H. Shintaku, S. Tanaka, S. Kawano, T. Kawai, and M. Taniguchi, Tracking singleparticle dynamics via combined optical and electrical sensing, Sci. Rep. 3, 1855 (2013).

[7] B. J. Sanghavi, W. Varhue, J. L. Chavez, C.-F. Chou, and N. S. Swami, Electrokinetic preconcentration and detection of neuropeptides at patterned graphene-modified electrodes in a nanochannel, Anal. Chem. 86, 4120 (2014).

[8] P. Arosio, K. Hu, F. A. Aprile, T. Muller, and T. P. J. Knowles, Microfluidic diffusion viscometer for rapid analysis of complex solutions, Anal. Chem. 88, 3488 (2016).

[9] K. M. Warren, J. N. Mpagazehe, P. R. LeDuc, and C. F. Higgs III, Geometric effects in microfluidics on heterogeneous cell stress using an eulerian-lagrangian approach, Lab Chip 16, 593 (2016).

[10] K. D. Nyberg, M. B. Scott, S. L. Bruce, A. B. Gopinath, D. Bikos, T. G. Mason, J. W. Kim, H. S. Choi, and A. C. Rowat, The physical origins of transit time measurements for rapid, single cell mechanotyping, Lab Chip 16, 3330 (2016).

[11] I. Hanasaki and J. H. Walther, Suspended particle transport through constriction channel with brownian motion, Phys. Rev. E 96, 023109 (2017).

[12] M. Tsutsui, Y. Maeda, Y. He, S. Hongo, S. Ryuzaki, S. Kawano, T. Kawai, and M. Taniguchi, Trapping and identifying single-nanoparticles using alow-aspect-ratio nanopore, Appl. Phys. Lett. 103, 013108 (2013).

[13] R. Piazza, Thermophoresis: Moving particles with thermal gradients, Soft Matter 4, 1740 (2008).

[14] R. Piazza and A. Parola, Thermophoresis in colloidal suspensions, J. Phys.: Condens. Matter 20, 153102 (2008).

[15] A. Würger, Thermal non-equilibrium transport in colloids, Rep. Prog. Phys. 73, 126601 (2010).

[16] S. Duhr and D. Braun, Why molecules move along a temperature gradient, Proc. Natl. Acad. Sci. USA 103, 19678 (2006).
[17] A. Würger, Molecular-Weight Dependent Thermal Diffusion in Dilute Polymer Solutions, Phys. Rev. Lett. 102, 078302 (2009).

[18] P. Reineck, C. J. Wienken, and D. Braun, Thermophoresis of single stranded DNA, Electrophoresis 31, 279 (2010).

[19] Y. T. Maeda, A. Buguin, and A. Libchaber, Thermal Separation: Interplay between the Soret Effect and Entropic Force Gradient, Phys. Rev. Lett. 107, 038301 (2011).

[20] S. A. I. Seidel, C. J. Wienken, S. Geissler, M. JerabekWillemsen, S. Duhr, A. Reiter, D. Trauner, D. Braun, and P. Baaske, Label-free microscale thermophoresis discriminates sites and affinity of protein-ligand binding, Angew. Chem. Int. Edit. 51, 10656 (2012).

[21] Y. T. Maeda, T. Tlusty, and A. Libchaber, Effects of long DNA folding and small RNA stem-loop in thermophoresis, Proc. Natl. Acad. Sci. USA 109, 17972 (2012).

[22] A. Ly and A. Würger, Hydrodynamic interactions in DNA thermophoresis, Soft Matter 14, 848 (2018).

[23] J. Chen, H. Cong, F.-C. Loo, Z. Kang, M. Tang, H. Zhang, S.-Y. Wu, S.-K. Kong, and H.-P. Ho, Thermal gradient induced tweezers for the manipulation of particles and cells, Sci. Rep. 6, 35814 (2016).

[24] R. Piazza, Thermal diffusion in ionic micellar solutions, Philos. Mag. 83, 2067 (2003).

[25] A. Regazzetti, M. Hoyos, and M. Martin, Experimental evidence of thermophoresis of non-brownian particles in pure liquids and estimation of their thermophoretic mobility, J. Phys. Chem. B 108, 15285 (2004).

[26] S. A. Putnam and D. G. Cahill, Transport of nanoscale latex spheres in a temperature gradient, Langmuir 21, 5317 (2005).

[27] S. A. Putnam, D. G. Cahill, and G. C. L. Wong, Temperature dependence of thermodiffusion in aqueous suspensions of charged nanoparticles, Langmuir 23, 9221 (2007).

[28] S. Iacopini, R. Rusconi, and R. Piazza, "The macromolecular tourist": Universal temperature dependence of thermal diffusion in aqueous colloidal suspensions, Euro. Phys. J. E 19, 59 (2006).

[29] M. Braibanti, D. Vigolo, and R. Piazza, Does Thermophoretic Mobility Depend on Particle Size? Phys. Rev. Lett. 100, 108303 (2008).

[30] H.-R. Jiang, H. Wada, N. Yoshinaga, and M. Sano, Manipulation of Colloids by a Nonequilibrium Depletion Force in a Temperature Gradient, Phys. Rev. Lett. 102, 208301 (2009).

[31] D. Vigolo, R. Rusconi, H. A. Stone, and R. Piazza, Thermophoresis: Microfluidics characterization and separation, Soft Matter 6, 3489 (2010).

[32] D. Vigolo, S. Buzzaccaro, and R. Piazza, Thermophoresis and thermoelectricity in surfactant solutions, Langmuir $\mathbf{2 6}$, 7792 (2010).

[33] K. A. Eslahian, A. Majee, M. Maskos, and A. Würger, Specific salt effects on thermophoresis of charged colloids, Soft Matter 10, 1931 (2014).

[34] T. Tsuji, K. Kozai, H. Ishino, and S. Kawano, Direct observations of thermophoresis in microfluidic systems, Micro Nano Lett. 12, 520 (2017).

[35] J. Burelbach, M. Zupkauskas, R. Lamboll, Y. Lan, and E. Eiser, Colloidal motion under the action of a thermophoretic force, J. Chem. Phys. 147, 094906 (2017). 
[36] L. Lin, X. Peng, Z. Mao, X. Wei, C. Xie, and Y. Zheng, Interfacial-entropy-driven thermophoretic tweezers, Lab Chip 17, 3061 (2017).

[37] L. Lin, X. Peng, X. Wei, Z. Mao, C. Xie, and Y. Zheng, Thermophoretic tweezers for low-power and versatile manipulation of biological cells, ACS Nano 11, 3147 (2017).

[38] L. Lin, J. Zhang, X. Peng, Z. Wu, A. C. H. Coughlan, Z. Mao, M. A. Bevan, and Y. Zheng, Opto-thermophoretic assembly of colloidal matter, Sci. Adv. 3, e1700458 (2017).

[39] T. Tsuji, S. Saita, and S. Kawano, Dynamic Pattern Formation of Microparticles in a Uniform Flow by an On-Chip Thermophoretic Separation Device, Phys. Rev. Appl. 9, 024035 (2018).

[40] L. Lin, M. Wang, X. Peng, E. N. Lissek, Z. Mao, L. Scarabelli, E. Adkins, S. Coskun, H. E. Unalan, B. A. Korgel et al., Opto-thermoelectric nanotweezers, Nat. Photonics 12, 195 (2018).

[41] S. Duhr and D. Braun, Optothermal Molecule Trapping by Opposing Fluid Flow with Thermophoretic Drift, Phys. Rev. Lett. 97, 038103 (2006).

[42] C. J. Wienken, P. Baaske, U. Rothbauer, D. Braun, and S. Duhr, Protein-binding assays in biological liquids using microscale thermophoresis, Nat. Commun. 1, 100 (2010).

[43] J. L. Parker and S. Newstead, Molecular basis of nitrate uptake by the plant nitrate transporter NRT1.1, Nature 507, 68 (2014).

[44] G. Galliéro and S. Volz, Thermodiffusion in model nanofluids by molecular dynamics simulations, J. Chem. Phys. 128, 064505 (2008).

[45] T. Tsuji, H. Iseki, I. Hanasaki, and S. Kawano, Molecular dynamics study of force acting on a model nano particle immersed in fluid with temperature gradient: Effect of interaction potential, AIP Conf. Proc. 1786, 110003 (2016).

[46] T. Tsuji, H. Iseki, I. Hanasaki, and S. Kawano, Negative thermophoresis of nanoparticles interacting with fluids through a purely-repulsive potential, J. Phys.: Condens. Matter 29, 475101 (2017).

[47] D. Lüsebrink, M. Yang, and M. Ripoll, Thermophoresis of colloids by mesoscale simulations, J. Phys.: Condens. Matter 24, 284132 (2012).

[48] J. L. Anderson, Colloid transport by interfacial forces, Annu. Rev. Fluid Mech. 21, 61 (1989).

[49] A. Würger, Thermophoresis in Colloidal Suspensions Driven by Marangoni Forces, Phys. Rev. Lett. 98, 138301 (2007).

[50] A. Würger, Transport in Charged Colloids Driven by Thermoelectricity, Phys. Rev. Lett. 101, 108302 (2008).

[51] T. Tsuji, S. Saita, and S. Kawano, Thermophoresis of a Brownian particle driven by inhomogeneous thermal fluctuation, Physica A 493, 467 (2018).

[52] J. Burelbach, D. Frenkel, I. Pagonabarraga, and E. Eiser, A unified description of colloidal thermophoresis, Euro. Phys. J. E 41, 7 (2018).

[53] S. M. Bezrukov, I. Vodyanoy, and V. A. Parsegian, Counting polymers moving through a single ion channel, Nature 370, 279 (1994).

[54] J. J. Kasianowicz, E. Brandin, D. Branton, and D. W. Deamer, Characterization of individual polynucleotide molecules using a membrane channel, Proc. Natl. Acad. Sci. USA 93, 13770 (1996).

[55] D. Branton, D. W. Deamer, A. Marziali, H. Bayley, S. A. Benner, T. Butler, M. Di Ventra, S. Garaj, A. Hibbs, X. Huang et al., The potential and challenges of nanopore sequencing, Nat. Biotechnol. 26, 1146 (2008).

[56] M. Tsutsui, M. Taniguchi, K. Yokota, and T. Kawai, Identifying single nucleotides by tunnelling current, Nat. Nanotechnol. 5, 286 (2010).

[57] G. F. Schneider, S. W. Kowalczyk, V. E. Calado, G. Pandraud, H. W. Zandbergen, L. M. K. Vandersypen, and C. Dekker, DNA translocation through graphene nanopores, Nano Lett. 10, 3163 (2010).

[58] C. A. Merchant, K. Healy, M. Wanunu, V. Ray, N. Peterman, J. Bartel, M. D. Fischbein, K. Venta, Z. Luo, A. T. C. Johnson et al., DNA translocation through graphene nanopores, Nano Lett. 10, 2915 (2010).

[59] D. Deamer, M. Akeson, and D. Branton, Three decades of nanopore sequencing, Nat. Biotechnol. 34, 518 (2016).

[60] U. F. Keyser, Controlling molecular transport through nanopores, J. Royal Soc. Interface 8, 1369 (2011).

[61] E. C. Yusko, J. M. Johnson, S. Majd, P. Prangkio, R. C. Rollings, J. Li, J. Yang, and M. Mayer, Controlling protein translocation through nanopores with bio-inspired fluid walls, Nat. Nanotechnol. 6, 253 (2011).

[62] W. Qian, K. Doi, S. Uehara, K. Morita, and S. Kawano, Theoretical study of the transpore velocity control of singlestranded DNA, Int. J. Mol. Sci. 15, 13817 (2014).

[63] S. E. Henrickson, M. Misakian, B. Robertson, and J. J. Kasianowicz, Driven DNA Transport into an Asymmetric Nanometer-Scale Pore, Phys. Rev. Lett. 85, 3057 (2000).

[64] J. Nakane, M. Wiggin, and A. Marziali, A nanosensor for transmembrane capture and identification of single nucleic acid molecules, Biophys. J. 87, 615 (2004).

[65] M. Rincon-Restrepo, E. Mikhailova, H. Bayley, and G. Maglia, Controlled translocation of individual DNA molecules through protein nanopores with engineered molecular brakes, Nano Lett. 11, 746 (2011).

[66] W. Qian, K. Doi, and S. Kawano, Effects of polymer length and salt concentration on the transport of SSDNA in nanofluidic channels, Biophys. J. 112, 838 (2017).

[67] S. Howorka, L. Movileanu, X. Lu, M. Magnon, S. Cheley, $\mathrm{O}$. Braha, and H. Bayley, A protein pore with a single polymer chain tethered within the lumen, J. Am. Chem. Soc. 122, 2411 (2000).

[68] M. Bates, M. Burns, and A. Meller, Dynamics of DNA molecules in a membrane channel probed by active control techniques, Biophys. J. 84, 2366 (2003).

[69] A. Meller, L. Nivon, and D. Branton, Voltage-Driven DNA Translocations through a Nanopore, Phys. Rev. Lett. 86, 3435 (2001).

[70] S. Tanaka, M. Tsutsui, H. Theodore, H. Yuhui, A. Arima, T. Tsuji, K. Doi, S. Kawano, M. Taniguchi, and T. Kawai, Tailoring particle translocation via dielectrophoresis in pore channels, Sci. Rep. 6, 31670 (2016).

[71] Y. He, M. Tsutsui, R. H. Scheicher, F. Bai, M. Taniguchi, and T. Kawai, Thermophoretic manipulation of DNA translocation through nanopores, ACS Nano 7, 538 (2012).

[72] M. Belkin, C. Maffeo, D. B. Wells, and A. Aksimentiev, Stretching and controlled motion of single-stranded DNA 
in locally heated solid-state nanopores, ACS Nano 7, 6816 (2013).

[73] M. Belkin, S.-H. Chao, G. Giannetti, and A. Aksimentiev, Modeling thermophoretic effects in solid-state nanopores, J. Comput. Electron. 13, 826 (2014).

[74] H. Yamazaki, R. Hu, R. Y. Henley, J. Halman, K. A. Afonin, D. Yu, Q. Zhao, and M. Wanunu, Label-free singlemolecule thermoscopy using a laser-heated nanopore, Nano Lett. 17, 7067 (2017).

[75] M. Zhang, C. Ngampeerapong, D. Redin, A. Ahmadian, I. Sychugov, and J. Linnros, Thermophoresis controlled size-dependent DNA translocation through an array of nanopores, ACS Nano 12, 4574 (2018).

[76] S.-W. Kang and D. Banerjee, Modeling and simulation of capillary microfluidic networks based on electrical analogies, J. Fluids Eng. 133, 054502 (2011).

[77] A.-J. Mäki, S. Hemmilä, J. Hirvonen, N. N. Girish, J. Kreutzer, J. Hyttinen, and P. Kallio, Modeling and experimental characterization of pressure drop in gravitydriven microfluidic systems, J. Fluids Eng. 137, 021105 (2015).

[78] E. D. Palik, Handbook of Optical Constants of Solids (Elsevier, Amsterdam, 1997).

[79] M. L. Cordero, E. Verneuil, F. Gallaire, and C. N. Baroud, Time-resolved temperature rise in a thin liquid film due to laser absorption, Phys. Rev. E 79, 011201 (2009).

[80] D. Rivière, B. Selva, H. Chraibi, U. Delabre, and J.-P. Delville, Convection flows driven by laser heating of a liquid layer, Phys. Rev. E 93, 023112 (2016).

[81] H. Bruus, Theoretical Microfluidics (Oxford University Press, Oxford, 2007).

[82] C. V. Nguyen, A. Fouras, and J. Carberry, Improvement of measurement accuracy in micro PIV by image overlapping, Exp. Fluids 49, 701 (2010).

[83] D. Braun and A. Libchaber, Trapping of DNA by Thermophoretic Depletion and Convection, Phys. Rev. Lett. 89, 188103 (2002).

[84] T. Shoji, M. Shibata, N. Kitamura, F. Nagasawa, M. Takase, K. Murakoshi, A. Nobuhiro, Y. Mizumoto, H. Ishihara, and Y. Tsuboi, Reversible photoinduced formation and manipulation of a two-dimensional closely packed assembly of polystyrene nanospheres on a metallic nanostructure, J. Phys. Chem. C 117, 2500 (2013).

[85] T. Shoji and Y. Tsuboi, Plasmonic optical tweezers toward molecular manipulation: Tailoring plasmonic nanostructure, light source, and resonant trapping, J. Phys. Chem. Lett. 5, 2957 (2014).

[86] T. Shoji and Y. Tsuboi, Plasmonic optical trapping of soft nanomaterials such as polymer chains and DNA: Micropatterning formation, Opt. Rev. 22, 137 (2015).

[87] K. Setoura, S. Ito, and H. Miyasaka, Stationary bubble formation and marangoni convection induced by $\mathrm{CW}$ laser heating of a single gold nanoparticle, Nanoscale 9, 719 (2016).

[88] R. Milo, P. Jorgensen, U. Moran, G. Weber, and M. Springer, Bionumbers-the database of key numbers in molecular and cell biology, Nucleic Acids Res. 38, D750 (2009).

[89] N. M. Bouvier and P. Palese, The biology of influenza viruses, Vaccine 26, D49 (2008).

[90] J. A. G. Briggs, K. Grünewald, B. Glass, F. Förster, H.-G. Kräusslich, and S. D. Fuller, The mechanism of HIV-1 core assembly: Insights from three-dimensional reconstructions of authentic virions, Structure 14, 15 (2006).

[91] J.-M. Claverie, H. Ogata, S. Audic, C. Abergel, K. Suhre, and P.-E. Fournier, Mimivirus and the emerging concept of "giant" virus, Virus Res. 117, 133 (2006).

[92] V. Lindroos, T. Motooka, S. Franssila, M. PaulastoKrockel, M. Tilli, and V.-M. Airaksinen, Handbook of Silicon Based MEMS Materials and Technologies (Elsevier, Amsterdam, 2015), 2nd ed.

[93] M. A. Green and M. J. Keevers, Optical properties of intrinsic silicon at $300 \mathrm{~K}$, Prog. Photovoltaics Res. Appl. 3, 189 (1995).

[94] B. E. A. Saleh, M. C. Teich, and B. E. Saleh, Fundamentals of Photonics (Wiley, New York, 1991), Vol. 22.

[95] Y. Harada and T. Asakura, Radiation forces on a dielectric sphere in the rayleigh scattering regime, Opt. Commun. 124, 529 (1996).

[96] A. Rohrbach and E. H. K. Stelzer, Optical trapping of dielectric particles in arbitrary fields, J. Opt. Soc. Am. A 18, 839 (2001). 